Document downloaded from:

http://hdl.handle.net/10251/61010

This paper must be cited as:

Archetti, C.; Speranza, MG.; Corberan, A.; Sanchís Llopis, JM.; Plana, I. (2014). The Team Orienteering Arc Routing Problem. Transportation Science. 48(3):442-457. doi:10.1287/trsc.2013.0484.

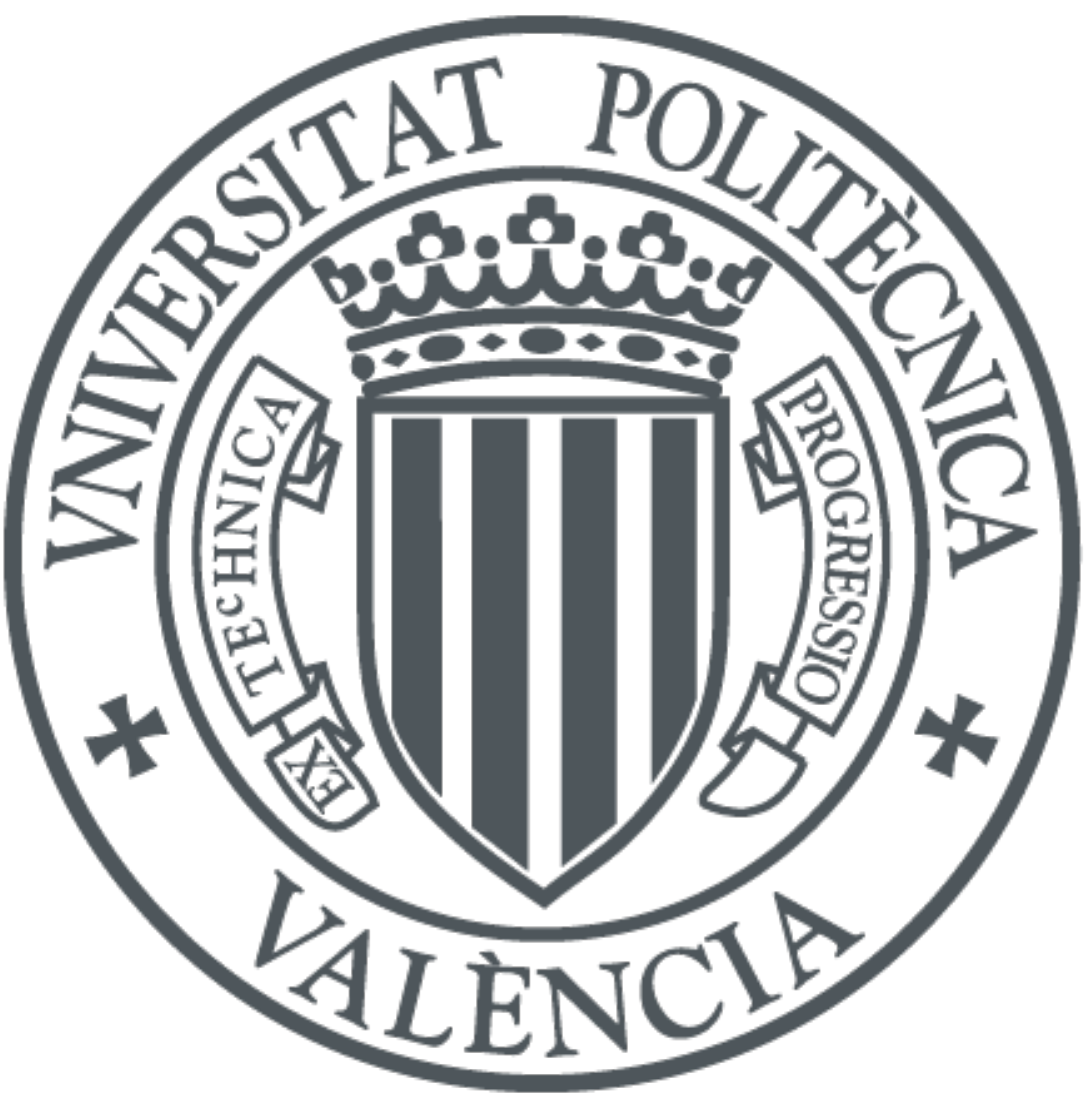

The final publication is available at

http://dx.doi.org/10.1287/trsc.2013.0484

Copyright INFORMS (Institute for Operations Research and Management Sciences)

Additional Information 


\title{
The Team Orienteering Arc Routing Problem
}

\author{
Claudia $\operatorname{Archetti}^{(1)} \quad$ Ángel Corberán ${ }^{(2)} \quad$ Isaac Plana ${ }^{(4)}$ \\ José M. Sanchis ${ }^{(3)}$ M. Grazia $\operatorname{Speranza}^{(1)}$ \\ (1) Dipartimento Metodi Quantitativi, Universitá di Brescia, Italy \\ (2) Departamento de Estadística e Investigación Operativa, Universidad de Valencia, Spain \\ (4) Departamento Matemáticas para la Economía y la Empresa, Universidad de Valencia, Spain \\ (3) Departamento de Matemática Aplicada, Universidad Politécnica de Valencia, Spain
}

\{archetti, speranza\}@eco.unibs.it, \{angel.corberan, isaac.plana\}@uv.es, jmsanchis@mat.upv.es

September 26, 2012

\begin{abstract}
The Team Orienteering Arc Routing Problem (TOARP) is the extension to the arc routing setting of the Team Orienteering Problem (TOP). In the TOARP, in addition to a possible set of regular customers that have to be serviced, another set of potential customers is available. Each customer is associated with an arc of a directed graph. Each potential customer has a profit that is collected when it is serviced, that is, when the associated arc is traversed. A fleet of vehicles with a given maximum traveling time is available. The profit from a customer can be collected by one vehicle at most. The objective is to identify the customers which maximize the total profit collected while satisfying the given time limit for each vehicle.

In this paper we propose a formulation for this problem and study its associated polyhedron. We present some families of valid and facet-inducing inequalities that we use in the implementation of a branch-and-cut algorithm for the resolution of the problem. Computational experiments are run on a large set of benchmark instances.
\end{abstract}

Keywords: Team Orienteering Arc Routing Problem, Profits, Multi-vehicle, Facets, Branch and Cut.

\section{Introduction}

A large number of papers have appeared in the literature studying routing problems where the set of customers to be visited (nodes or arcs) is given a priori. In the situations modeled, all customers to be visited are known at the time the optimization model is run. While this is indeed the case in many practical problems, there are several other different practical situations. For example, not all customers may need to be visited and instead have to be selected from a set. In this paper we focus on an arc routing problem.

Let us consider the following situation. Nowadays it is more and more frequent for demand for transportation services to be posted on the web, usually in specific databases, and carriers

${ }^{*}$ corresponding author 
can respond to that demand and offer their services to some of these customers, possibly in the framework of an electronic auction. Thus, from within a set of potential customers, the carrier has to select those which are most convenient for him. In an electronic auction, the carrier will make a bid for these potential customers. In a truck-load type of service, a transportation service consists of reaching a node with an empty truck, filling the truck with a load, traversing an arc and unloading the truck completely. A fleet of vehicles with limited traveling time is usually available to perform the service. The carrier may need to take into account a set of customers who need to be serviced because the carrier has regular contract with them. This is, in fact, the most common situation. The carrier looks for additional potential customers in order to fully use the unused traveling time of some of the vehicles.

The situation described above can be modeled as an arc routing problem with profits. Given a directed graph, a profit is associated with each arc of a subset of the arcs (the arcs which represent potential customers). A traversing time is associated with each of the arcs of the graph. A fleet of vehicles, each with a limited traveling time, is available to perform the service. The objective is to maximize the profit collected.

While a good number of papers are available on node routing problems with profits (see the surveys [13] and [21]), very few are available on arc routing problems with profits. Malandraki and Daskin [18] introduce the Maximum Benefit Chinese Postman Problem (MBCPP) on a directed graph. Basically, in this problem several profits are associated with each arc, one for each time the arc is traversed with a service, and the objective is to find a closed walk with maximum profit. In [19] and [20], some heuristic algorithms for solving the MBCPP on directed and undirected graphs are proposed, while in [10], the polyhedron associated with the undirected MBCPP is studied and a branch-and-cut algorithm is proposed.

The Prize-Collecting Arc Routing Problem (PCARP), also called Privatized Rural Postman Problem, is a special case of the MBCPP in which only the edges in a given subset have an associated benefit that can be collected just once. The PCARP is introduced in [4], where an ILP formulation with binary variables is provided. In [3], an LP-based algorithm for solving the problem on undirected graphs is proposed. A related problem, the Clustered Prize-collecting Arc Routing Problem, is studied in [2] for undirected graphs and in [9] for 'windy' graphs. Other arc routing problems with benefits, such as the Profitable Arc Tour Problem and the Undirected Capacitated Arc Routing Problem with Profits, are studied in [14] and [6]. This last paper is the first one devoted to a multi-vehicle arc routing problem with profits.

In this paper, we introduce another multi-vehicle arc routing problem with profits, the TOARP. We study its associated polyhedron and propose a branch-and-cut algorithm for its exact resolution. The remainder of the paper is organized as follows. In Section 2 we define the problem and model it as an integer program. Sections 3 and 4 are devoted to the polyhedral study of the problem. The proposed branch-and-cut algorithm is presented in Section 5, while the computational experiments are described in Section 6. Finally, in Section 7 some conclusions are drawn.

\section{The Team Orienteering Arc Routing Problem}

We consider a directed graph $G=(V, A)$, where $V=\{1, \ldots, n\}$ is the set of vertices and $A$ is the set of arcs. Vertex 1 is the starting and ending point of each tour. A subset $A_{R}$ of $A$ represents customers that have to be serviced obligatorily, while $A^{\prime} \subseteq A$ represents the set of potential customers. A nonnegative profit $s_{i j}$ is associated with each arc $(i, j) \in A^{\prime}$. A traveling time $t_{i j}$ is associated with each $\operatorname{arc}(i, j) \in A$. A set of $K$ vehicles is available 
to service the customers. Each vehicle can visit any subset of the obligatory and potential customers within a given time limit $T_{\max }$. The profit from each potential customer can be collected only once and by one vehicle at most. The objective of the Team Orienteering Arc Routing Problem (TOARP) is to maximize the total profit collected while satisfying the time limit $T_{\max }$ for each vehicle.

In this paper it is assumed, without loss of generality, that all the vertices are incident with arcs in $A_{R} \cup A^{\prime}$. It can be seen that if $G$ does not satisfy this condition, it can be transformed into an equivalent graph which does.

We use the following notation. Given a subset of vertices $S \subseteq V$, let $A(S)$ be the set of arcs with both endpoints in $S, A(S)=\{(i, j) \in A: i, j \in S\}$. Similarly for $A^{\prime}(S)$ and $A_{R}(S)$. The subgraph of $G$ induced by the set of vertices $S$ is denoted by $G(S)$. Given two disjoint sets $S, T \subseteq V$, we define $(S, T)=\{(i, j) \in A: i \in S, j \in T\}$ and $(S: T)=(S, T) \cup(T, S)$, and $\delta(S)=(S: V \backslash S)$. Finally, given a set of arcs $W \subset A$ and a vector $x$ indexed by the $\operatorname{arcs}$ in $A, x(W)=\sum_{a \in W} x_{a}$.

We can formulate this problem by defining the following variables:

- For each $(i, j) \in A$ and each vehicle $k=1, \ldots, K$, let $x_{i j}^{k}$ be the number of times that vehicle $k$ traverses arc $(i, j)$.

- For each $(i, j) \in A_{R} \cup A^{\prime}$ and each vehicle $k=1, \ldots, K$, let $y_{i j}^{k}$ be a binary variable that takes value 1 if vehicle $k$ services the customer $(i, j)$, and value 0 otherwise.

The corresponding model is:

$$
\text { Maximize } \quad \sum_{k=1}^{K} \sum_{(i, j) \in A^{\prime}} s_{i j} y_{i j}^{k}
$$

s.t.:

$$
\begin{aligned}
\sum_{j \in V \backslash\{i\}} x_{i j}^{k}=\sum_{j \in V \backslash\{i\}} x_{j i}^{k} & \forall i \in V, \quad k=1, \ldots, K \\
\sum_{i \in V \backslash S, j \in S} x_{i j}^{k} \geq y_{a}^{k} & \forall S \subset V \backslash\{1\}, \quad \forall a \in A^{\prime}(S) \cup A_{R}(S), \quad k=1, \ldots, K \\
x_{i j}^{k} \geq y_{i j}^{k} & \forall(i, j) \in A^{\prime} \cup A_{R}, \quad k=1, \ldots, K \\
\sum_{k=1}^{K} y_{i j}^{k}=1 & \forall(i, j) \in A_{R} \\
\sum_{k=1}^{K} y_{i j}^{k} \leq 1 & \forall(i, j) \in A^{\prime} \\
\sum_{(i, j) \in A} t_{i j} x_{i j}^{k} \leq T_{\text {max }} & k=1, \ldots, K \\
x_{i j}^{k} \geq 0 \text { and integer } & \forall(i, j) \in A, \quad k=1, \ldots, K \\
y_{i j}^{k} \in\{0,1\} & \forall(i, j) \in A^{\prime} \cup A_{R}, \quad k=1, \ldots, K,
\end{aligned}
$$

where (1) are the symmetry equations, connectivity constraints (2) ensure that each route servicing at least one arc is connected and goes through the depot, constraints (3) force each vehicle to traverse the arcs that it services, constraints (4) guarantee that each required arc 
in $A_{R}$ is serviced by exactly one vehicle, constraints (5) ensure that the profit from each potential customer is collected by at most one vehicle, and constraints (6) limit the length or time of each route.

\section{The TOARP polyhedron}

As with the Capacitated Vehicle Routing Problem polytope or the Capacitated Arc Routing Problem polyhedron, determining the dimension of the polyhedron defined as the convex hull of the TOARP solutions is a very difficult task, because it does not depend only on the number of vertices and arcs, but also on the arc lengths $t_{i j}$, the number of vehicles $K$, and the time limit $T_{\max }$. In some cases, the dimension could even be zero. The difficulty in obtaining the dimension makes the task of proving that an inequality is facet-inducing for the polyhedron almost impossible in general. However, if we remove the constraints (6) that limit the length of each route, the resulting relaxed polyhedron can indeed be studied. We think this is interesting because some of its facets can also be facets of the original TOARP polyhedron and it is a way of guaranteeing the tightness of the constraints in the formulation and of the valid inequalities we can find. Note that constraints (6) have the effect of cutting off part of the relaxed polyhedron. The size of the part of the polyhedron that is cut off depends on how tight the time limit for the routes is.

In what follows, we study the relaxed polyhedron defined by the convex hull of the vectors $\left(x^{1}, y^{1}, x^{2}, y^{2}, \ldots, x^{K}, y^{K}\right)$ with $\left(|A|+\left|A_{R}\right|+\left|A^{\prime}\right|\right) K$ components satisfying (1) to (5), (7), and (8). We call such a vector a TOARP solution, although it can violate the time limit constraints, and denote the resulting polyhedron by $\mathcal{P}$. Note that solutions where a vehicle tour $x^{k}$ is formed of several disconnected subtours, one of them connecting all the arcs it services to the depot and the others traversing arcs not serviced by this vehicle are then permitted. This property is needed for the convex hull of the TOARP solutions to be a polyhedron. Note also that solutions where a given vehicle neither traverses nor services any arcs are also permitted.

In this section we study the dimension of $\mathcal{P}$ and prove that most of the inequalities in the formulation define facets of it. In the following theorems we assume that $A_{R} \neq \emptyset$. The proof for the cases in which $A_{R}=\emptyset$ is similar.

Theorem 1 If $G$ is strongly connected then, for all $K \geq 1$, the dimension of $\mathcal{P}$ is $(|A|+$ $\left.\left|A^{\prime}\right|-|V|+1\right) K+\left|A_{R}\right|(K-1)$.

Proof: It can be proved that $\mathcal{P}$ is a polyhedron in $\mathbb{Z}^{K\left(|A|+\left|A_{R}\right|+\left|A^{\prime}\right|\right)}$. All its points satisfy the $\left|A_{R}\right|$ linearly independent equations (4) and the $K|V|$ equations (1), $K|V-1|$ of which are linearly independent. Therefore, $\operatorname{dim}(\mathcal{P}) \leq\left(|A|+\left|A^{\prime}\right|-|V|+1\right) K+\left|A_{R}\right|(K-1)$.

To prove the equality, we have to find $\left(|A|+\left|A^{\prime}\right|-|V|+1\right) K+\left|A_{R}\right|(K-1)+1$ affinely independent TOARP solutions (or linearly independent, because $0 \notin \operatorname{aff}(\mathcal{P})$ if $A_{R} \neq \emptyset$ ).

Consider the (one vehicle) Directed General Routing Problem (DGRP) defined on the graph $G^{*}=(V, A)$, where the set of required arcs is given by $A_{R}^{*}=A_{R} \cup A^{\prime}$ and all the vertices in $V$ are required. Remember that the DGRP consists of finding a minimum cost tour on a directed graph traversing each required arc and visiting each required vertex at least once. If $\operatorname{DGRP}\left(G^{*}\right)$ denotes the associated polyhedron and given that the DGRP is a special case of the Mixed General Routing Problem (MGRP), with $E=\emptyset$, we have $\operatorname{dim}\left(\operatorname{DGRP}\left(G^{*}\right)\right)=|A|-|V|+1[11]$. 
Let us denote $m=\operatorname{dim}\left(\operatorname{DGRP}\left(G^{*}\right)\right)$. Since $0 \in \operatorname{aff}\left(\operatorname{DGRP}\left(G^{*}\right)\right)$, there are $m$ linearly independent DGRP tours $z_{1}, z_{2}, \ldots, z_{m}$ on $G^{*}$ which traverse all the arcs in $A_{R} \cup A^{\prime}$ and visit all the vertices in $V$ (and the depot in particular) at least once. From each DGRP tour, such as $z_{1}$, we can build the following TOARP solution for vehicle 1 :

- $x^{1}=z_{1}$, and $y_{a}^{1}=1 \quad \forall a \in A_{R}$ and $y_{a}^{1}=0 \quad \forall a \in A^{\prime}$,

- $x^{2}=\cdots=x^{K}=0$ and $y_{a}^{2}=\cdots=y_{a}^{K}=0 \quad \forall a \in A_{R} \cup A^{\prime}$,

in which vehicle 1 traverses all the arcs in $A_{R} \cup A^{\prime}$ as is done by $z_{1}$, services all the arcs in $A_{R}$ but none in $A^{\prime}$, and the remaining vehicles do nothing. This is the first row of the matrix in Figure 1, where for the sake of simplicity we suppose we have $K=3$ vehicles. A similar TOARP solution can be defined from each DGRP tour $z_{i}, i=1, \ldots, m$, thus obtaining the first block row of the matrix. The same procedure can be repeated for each vehicle $k=1, \ldots, K$. These $m \times K$ TOARP solutions are represented in the first 3 rows of blocks of the matrix in Figure 1, where a block with a large 0 or 1 represents a submatrix with all its entries 0 or 1 , respectively.

In the TOARP solutions represented in the last 3 rows of blocks of the matrix in Figure 1 , each vehicle $k$ traverses all the $\operatorname{arcs}$ in $A_{R} \cup A^{\prime}$ as is done by $z_{1}$; for example, it services all the $\operatorname{arcs}$ in $A_{R}$ plus exactly one arc in $A^{\prime}$. Here, a block with a large I represents an identity submatrix.

In the 4th and 5th rows of blocks, two different vehicles traverse all the $\operatorname{arcs}$ in $A_{R} \cup A^{\prime}$ and jointly service the arcs in $A_{R}$. Row 6 represents a TOARP solution in which all the arcs in $A_{R} \cup A^{\prime}$ are traversed by each one of the 3 vehicles. One of them services the first required arc and another one services the remaining arcs in $A_{R}$. These $\left(|A|+\left|A^{\prime}\right|-|V|+\right.$ 1) $K+\left|A_{R}\right|(K-1)+1$ TOARP solutions are linearly independent because the matrix has full rank. Note that from the structure of the matrix we can assert that the result is also true for any value of $K \geq 2$.

In the following we assume that graph $G$ is strongly connected. Moreover, as in the above theorem, in the proof of the theorems in this section, the matrices will be represented for the case with $K=3$ vehicles, although the results are valid for any value of $K$.

Theorem 2 If $G \backslash\{(i, j)\}$ is strongly connected, then inequalities $x_{i j}^{k} \geq 0$ are facet-inducing for $\mathcal{P}$ for all $(i, j) \in A \backslash\left(A_{R} \cup A^{\prime}\right)$ and for all $k$.

Proof: Let us consider, for example, $x_{i j}^{1} \geq 0$. We will find $d=\operatorname{dim}(\mathcal{P})$ affinely independent tours satisfying $x_{i j}^{1}=0$. Since $G \backslash\{(i, j)\}$ is strongly connected, $x_{i j} \geq 0$ is facet-inducing for $\operatorname{DGRP}\left(G^{*}\right)$ (see [11]), where $G^{*}=(V, A)$ is the graph with the set of required arcs $A_{R}^{*}=A_{R} \cup A^{\prime}$ and all the vertices in $V$ are required. Hence, there are $m=|A|-|V|+1$ affinely independent tours $w_{1}, \ldots, w_{m}$ satisfying $x_{i j}=0$. Let us assume that $w_{1}, \ldots, w_{m-1}$ are linearly independent. Furthermore, let $z_{1}, z_{2}, \ldots, z_{m}$ be the $m$ DGRP tours on $G^{*}$ defined in the proof of Theorem 1. Then the TOARP solutions written as rows in the matrix in Figure 2 prove the result.

Note 1 If $(i, j) \in A_{R} \cup A^{\prime}$, then $x_{i j}^{k} \geq 0$ is not facet-inducing for $\mathcal{P}$ because it is dominated by the corresponding inequality (3) $x_{i j}^{k} \geq y_{i j}^{k}$.

Theorem 3 If $G \backslash\{(i, j)\}$ is strongly connected, then inequalities $y_{i j}^{k} \geq 0$ are facet-inducing for $\mathcal{P}$ for all $(i, j) \in A_{R} \cup A^{\prime}$ and for all $k$. 


\begin{tabular}{|c|c|c|c|c|c|c|c|c|}
$x^{1}$ & $x^{2}$ & $x^{3}$ & $y_{A_{R}}^{1}$ & $y_{A_{R}}^{2}$ & $y_{A_{R}}^{3}$ & $y_{A^{\prime}}^{1}$ & $y_{A^{\prime}}^{2}$ & $y_{A^{\prime}}^{3}$ \\
\hline \hline $\begin{array}{c}z_{1} \\
\vdots \\
z_{m}\end{array}$ & 0 & 0 & 1 & 0 & 0 & 0 & 0 & 0 \\
\hline 0 & $\begin{array}{c}z_{1} \\
\vdots \\
z_{m}\end{array}$ & 0 & 0 & 1 & 0 & 0 & 0 & 0 \\
\hline 0 & 0 & $\begin{array}{c}z_{1} \\
\vdots \\
z_{m}\end{array}$ & 0 & 0 & 1 & 0 & 0 & 0 \\
\hline $\begin{array}{c}z_{1} \\
\vdots \\
z_{1}\end{array}$ & $\begin{array}{c}z_{1} \\
\vdots \\
z_{1}\end{array}$ & 0 & $\mathrm{I}$ & $1-\mathrm{I}$ & 0 & 0 & 0 & 0 \\
\hline 0 & $\begin{array}{c}z_{1} \\
\vdots \\
z_{1}\end{array}$ & $\begin{array}{c}z_{1} \\
\vdots\end{array}$ & 0 & $\mathrm{I}$ & $1-\mathrm{I}$ & 0 & 0 & 0 \\
\hline $\begin{array}{c}z_{1} \\
\vdots\end{array}$ & $\begin{array}{c}z_{1} \\
z_{1}\end{array}$ & 0 & $10 \ldots 0$ & $01 \ldots 1$ & 0 & 0 & 0 & 0 \\
\hline 0 & $\begin{array}{c}z_{1} \\
\vdots \\
z_{1}\end{array}$ & 0 & 0 & 1 & 0 & 0 & $\mathrm{I}$ & 0 \\
\hline 0 & 0 & $\begin{array}{c}z_{1} \\
\vdots\end{array}$ & 0 & 0 & 1 & 0 & 0 & $\mathrm{I}$ \\
\hline \hline
\end{tabular}

Figure 1: TOARP solutions to prove dimension.

Proof: For the $\operatorname{arcs}(i, j) \in A_{R}$, the proof is similar to that of Theorem 2 and is omitted here. For the $\operatorname{arcs}(i, j) \in A^{\prime}$, note that in the matrix shown in Figure 1 there is only a nonzero element in the column corresponding to $\operatorname{arc}(i, j)$ and vehicle $k$. If we remove the row containing this non-zero element, we obtain a full rank matrix whose vector rows represent TOARP solutions satisfying $y_{i j}^{k}=0$.

Note 2 Inequalities $y_{i j}^{k} \leq 1$ are not facet-inducing for $\mathcal{P}$ because they are dominated by inequalities $y_{i j}^{k} \geq 0$ and inequalities (4) or (5), either if $(i, j) \in A_{R}$ or $(i, j) \in A^{\prime}$, respectively.

Theorem 4 Inequalities $(3) x_{i j}^{k} \geq y_{i j}^{k}$ are facet-inducing for $\mathcal{P}$ for all $(i, j) \in A_{R} \cup A^{\prime}$ and for all $k$ if $G \backslash\{(i, j)\}$ is strongly connected.

Proof: Let us consider, for example, $x_{i j}^{1} \geq y_{i j}^{1}$. We will find $d=\operatorname{dim}(\mathcal{P})$ affinely independent tours satisfying $x_{i j}^{1}=y_{i j}^{1}$. Again, since $G \backslash\{(i, j)\}$ is strongly connected, $x_{i j} \geq 1$, for each $(i, j) \in A_{R} \cup A^{\prime}$, is facet-inducing for $\operatorname{DGRP}\left(G^{*}\right)$ (see [11]). Hence, there are $m=|A|-|V|+1$ affinely independent (and linearly independent, because 0 is not in the affine hull of the facet) tours $w_{1}, \ldots, w_{m}$ satisfying $x_{i j}=1$. Then, from these tours we can construct the TOARP solutions shown in Figures 3 and 4 for the case $(i, j) \in A_{R}$ and $(i, j) \in A^{\prime}$ respectively, where the columns corresponding to arc $(i, j)$ are the first ones in each block. It can be seen that the rank of both matrices is $d-1$.

Theorem 5 Inequalities $(5) \sum_{k=1}^{K} y_{i j}^{k} \leq 1$, for all $(i, j) \in A^{\prime}$, are facet-inducing for $\mathcal{P}$.

Proof: We will find $d=\operatorname{dim}(\mathcal{P})$ affinely independent tours satisfying $\sum_{k=1}^{K} y_{i j}^{k}=1$. Let $z_{1}, z_{2}, \ldots, z_{m}$ be the $m$ DGRP tours on $G^{*}$ defined in the proof of Theorem 1 . Then, from 


\begin{tabular}{|c|c|c|c|c|c|c|c|c|}
$x^{1}$ & $x^{2}$ & $x^{3}$ & $y_{A_{R}}^{1}$ & $y_{A_{R}}^{2}$ & $y_{A_{R}}^{3}$ & $y_{A^{\prime}}^{1}$ & $y_{A^{\prime}}^{2}$ & $y_{A^{\prime}}^{3}$ \\
\hline \hline $\begin{array}{c}w_{1} \\
\vdots \\
w_{m-1}\end{array}$ & 0 & 0 & 1 & 0 & 0 & 0 & 0 & 0 \\
\hline 0 & $\begin{array}{c}z_{1} \\
\vdots \\
z_{m}\end{array}$ & 0 & 0 & 1 & 0 & 0 & 0 & 0 \\
\hline 0 & 0 & $\begin{array}{c}z_{1} \\
\vdots \\
z_{m}\end{array}$ & 0 & 0 & 1 & 0 & 0 & 0 \\
\hline $\begin{array}{c}w_{1} \\
\vdots \\
w_{1}\end{array}$ & $\begin{array}{c}z_{1} \\
\vdots \\
z_{1}\end{array}$ & 0 & $\mathrm{I}$ & $1-\mathrm{I}$ & 0 & 0 & 0 & 0 \\
\hline 0 & $\begin{array}{c}z_{1} \\
\vdots \\
z_{1}\end{array}$ & $\begin{array}{c}z_{1} \\
\vdots\end{array}$ & 0 & $\mathrm{I}$ & $1-\mathrm{I}$ & 0 & 0 & 0 \\
\hline $\begin{array}{c}w_{1} \\
w_{1} \\
\vdots \\
w_{1}\end{array}$ & $\begin{array}{c}z_{1} \\
z_{1}\end{array}$ & 0 & $10 \ldots 0$ & $01 \ldots 1$ & 0 & 0 & 0 & 0 \\
\hline 0 & $\begin{array}{c}z_{1} \\
\vdots \\
z_{1}\end{array}$ & 0 & 0 & 1 & 0 & 0 & $\mathrm{I}$ & 0 \\
\hline 0 & 0 & $\begin{array}{c}z_{1} \\
\vdots\end{array}$ & 0 & 0 & 1 & 0 & 0 & $\mathrm{I}$ \\
\hline \hline
\end{tabular}

Figure 2: TOARP solutions satisfying $x_{i j}^{1}=0$.

these tours we can construct the TOARP solutions shown in Figure 5, where the columns corresponding to arc $(i, j)$ are the first in each block. It can be seen that the rank of this matrix is $d$. Note that the first row in the rows of blocks 1,2 and 3 is the same as the first one in the last three rows of blocks.

Theorem 6 Inequalities (2), $\sum_{i \in V \backslash S, j \in S} x_{i j}^{k} \geq y_{a}^{k}$, are facet-inducing for $\mathcal{P}$ for all $S \subset$ $V \backslash\{1\}$, for all $a \in A^{\prime}(S) \cup A_{R}(S)$ and for all $k \in\{1, \ldots, K\}$ if subgraphs $G(S)$ and $G(V \backslash S)$ are strongly connected.

Proof: Let $S \subset V \backslash\{1\}$ and $a \in A^{\prime}(S) \cup A_{R}(S)$. Let us consider, for example, $k=1$ and $a \in A^{\prime}(S)$ (the case $a \in A_{R}(S)$ is similar). We will find $d=\operatorname{dim}(\mathcal{P})$ affinely independent tours satisfying $\sum_{i \in V \backslash S, j \in S} x_{i j}^{1}=y_{a}^{1}$. Let $z_{1}, z_{2}, \ldots, z_{m}$ be the $m$ linearly independent DGRP tours on $G^{*}$ defined in the proof of Theorem 1. We define a DGRP instance $G^{\prime}$ in which all the arcs in $A$ are required except those in $\delta(S)$ and the depot is a required vertex. Given that subgraphs $G(S)$ and $G(V \backslash S)$ are strongly connected, inequality $\sum_{i \in V \backslash S, j \in S} x_{i j} \geq 1$ is facet-inducing for $\operatorname{DGRP}\left(G^{\prime}\right)$ (see [11]) and there are $m$ affinely and linearly independent tours $w_{1}, w_{2}, \ldots, w_{m}$ for the DGRP on $G^{\prime}$ satisfying $\sum_{i \in V \backslash S, j \in S} x_{i j}=1$. Note that these tours traverse only two arcs in $\delta(S)$ and therefore they are not able to service all the arcs in $\delta(S) \cap A_{R}$. Nevertheless, it can be seen that given any arc $f \in A^{\prime} \cup A_{R} \backslash\{a\}$, there is a tour $w_{i}$ above that traverses arcs $a$ and $f$ (the fourth and fifth rows of blocks in the matrix in Figure 6). It can be seen that the $d$ vectors represented in the matrix in Figure 6 are linearly independent feasible solutions for the TOARP and satisfy $\sum_{i \in V \backslash S, j \in S} x_{i j}^{1}=y_{a}^{1}$. 


\begin{tabular}{|c|c|c|c|c|c|c|c|c|}
\hline$x^{1}$ & $x^{2}$ & $\begin{array}{l}x^{3} \\
\end{array}$ & $y_{A_{R}}^{1}$ & $y_{A_{R}}^{2}$ & $y_{A_{R}}^{3}$ & $y_{A^{\prime}}^{1}$ & $y_{A^{\prime}}^{2}$ & $y_{A^{\prime}}^{3}$ \\
\hline $\begin{array}{c}w_{1} \\
\vdots \\
w_{m}\end{array}$ & 0 & 0 & 1 & 0 & 0 & 0 & 0 & 0 \\
\hline 0 & $\begin{array}{c}w_{1} \\
\vdots \\
w_{m}\end{array}$ & 0 & 0 & 1 & 0 & 0 & 0 & 0 \\
\hline 0 & 0 & $\begin{array}{c}w_{1} \\
\vdots \\
w_{m} \\
\end{array}$ & 0 & 0 & 1 & 0 & 0 & 0 \\
\hline $\begin{array}{c}w_{1} \\
\vdots \\
w_{1} \\
\end{array}$ & 0 & 0 & 1 & 0 & 0 & I & 0 & 0 \\
\hline 0 & $\begin{array}{c}w_{1} \\
\vdots \\
w_{1}\end{array}$ & 0 & 0 & 1 & 0 & 0 & I & 0 \\
\hline 0 & 0 & $\begin{array}{c}w_{1} \\
\vdots \\
w_{1}\end{array}$ & 0 & 0 & 1 & 0 & 0 & I \\
\hline $\begin{array}{c}w_{1} \\
\vdots \\
w_{1} \\
\end{array}$ & $\begin{array}{c}w_{1} \\
\vdots \\
w_{1} \\
\end{array}$ & 0 & $\begin{array}{ll}1 & \\
\vdots & 1-I \\
1 & \\
\end{array}$ & \begin{tabular}{|ll}
0 & \\
$\vdots$ & $\mathrm{I}$ \\
0 & \\
\end{tabular} & 0 & 0 & 0 & 0 \\
\hline 0 & $\begin{array}{c}w_{1} \\
\vdots \\
w_{1}\end{array}$ & $\begin{array}{c}w_{1} \\
\vdots \\
w_{1}\end{array}$ & 0 & 1-I & I & 0 & 0 & 0 \\
\hline 0 & $w_{1}$ & $w_{1}$ & 0 & $\begin{array}{lllllll}0 & 0 & 1 & \ldots & 1 \\
\end{array}$ & $110 \ldots 0$ & 0 & 0 & 0 \\
\hline
\end{tabular}

Figure 3: TOARP solutions satisfying $x_{i j}^{1}=y_{i j}^{1}$ for $(i, j) \in A_{R}$.

\section{More valid inequalities}

In this section, we present several families of valid inequalities for the TOARP, the K-C, path-bridge, and max-length inequalities.

\subsection{Path-bridge inequalities}

Path-bridge (PB) inequalities were introduced by Letchford [17] for the undirected General Routing Problem (GRP) and are based on the path inequalities for the Graphical Traveling Salesman Problem proposed by Cornuéjols, Fonlupt, and Naddef [12]. They were extended to the MGRP and, hence, to the DGRP in [11]. We first describe the PB inequalities for the DGRP and then we will extend them to the TOARP.

Let $G=(V, A)$ be a directed graph. Let us suppose that we have a subset $A_{R} \subset A$ of required arcs and that all the vertices in $V$ are also required. Let $P \geq 1$ and $B \geq 0$ be integers such that $P+B \geq 3$ is an odd number, and let $n_{1}, n_{2}, \ldots, n_{P}$ be integer numbers greater than or equal to 2 . Let us define a partition $\left\{M_{A}, M_{Z},\left\{M_{q}^{s}\right\}_{q=1, \ldots, n_{s}}^{s=1, \ldots, P}\right\}$ of $V$ satisfying that all the required arcs are contained either in $M_{A} \cup M_{Z}$ or in a set $M_{q}^{s}$ and $\left(M_{A}, M_{Z}\right)$ contains a number $B$ of required arcs. For the sake of simplicity we identify $M_{A}$ with $M_{0}^{s}$ and $M_{Z}$ with $M_{n_{s}+1}^{s}$ for all $s$. Hence, a PB inequality is defined by $P$ paths from $M_{A}$ to $M_{Z}$, each of them with $n_{s}+2$ node-sets, and by $B$ required $\operatorname{arcs}$ joining the sets $M_{A}$ and $M_{Z}$, which form the bridge. This configuration is shown in Figure 7, where the number near each couple of arcs joining sets $M_{q}^{s}, M_{r}^{t}$ represents the coefficient $b_{i j}$ associated with the variables in the PB inequality corresponding to all the $\operatorname{arcs}(i, j) \in\left(M_{q}^{s}, M_{r}^{t}\right)$. These coefficients are $b_{i j}=c\left(M_{q}^{s}, M_{r}^{t}\right)$, where:

$$
\text { - } c\left(M_{A}, M_{Z}\right)=c\left(M_{Z}, M_{A}\right)=1 \text {, }
$$




\begin{tabular}{|c|c|c|c|c|c|c|c|c|}
\hline$x^{1}$ & $x^{2}$ & $x^{3}$ & $y_{A_{R}}^{1}$ & $y_{A_{R}}^{2}$ & $y_{A_{R}}^{3}$ & $y_{A^{\prime}}^{1}$ & $y_{A^{\prime}}^{2}$ & $y_{A^{\prime}}^{3}$ \\
\hline $\begin{array}{c}w_{1} \\
\vdots \\
w_{m}\end{array}$ & 0 & 0 & 1 & 0 & 0 & $\begin{array}{cc}1 & \\
\vdots & 0 \\
1 & 0\end{array}$ & 0 & 0 \\
\hline 0 & $\begin{array}{c}w_{1} \\
\vdots \\
w_{m}\end{array}$ & 0 & 0 & 1 & 0 & 0 & 0 & 0 \\
\hline 0 & 0 & $\begin{array}{c}w_{1} \\
\vdots \\
w\end{array}$ & 0 & 0 & 1 & 0 & 0 & 0 \\
\hline $\begin{array}{c}w_{1} \\
\vdots \\
w_{1}\end{array}$ & 0 & 0 & 1 & 0 & 0 & $\begin{array}{l}10 \ldots 0 \\
\vdots \\
1\end{array}$ & 0 & 0 \\
\hline 0 & $\begin{array}{c}w_{1} \\
\vdots \\
w_{1}\end{array}$ & 0 & 0 & 1 & 0 & 0 & I & 0 \\
\hline 0 & 0 & $\begin{array}{c}w_{1} \\
\vdots \\
w_{1}\end{array}$ & 0 & 0 & 1 & 0 & 0 & I \\
\hline $\begin{array}{c}w_{1} \\
\vdots \\
w_{1}\end{array}$ & $\begin{array}{c}w_{1} \\
\vdots \\
w_{1}\end{array}$ & 0 & I & 1-I & 0 & $\begin{array}{ll}1 & \\
\vdots & 0 \\
1 & 0\end{array}$ & 0 & 0 \\
\hline 0 & $\begin{array}{c}w_{1} \\
\vdots \\
w_{1}\end{array}$ & $\begin{array}{c}w_{1} \\
\vdots \\
w_{1}\end{array}$ & 0 & I & $1-\mathrm{I}$ & 0 & 0 & 0 \\
\hline$w_{1}$ & $w_{1}$ & $w_{1}$ & $10 \ldots 0$ & $01 \ldots 1$ & 0 & $10 \ldots 0$ & 0 & 0 \\
\hline
\end{tabular}

Figure 4: TOARP solutions satisfying $x_{i j}^{1}=y_{i j}^{1}$ for $(i, j) \in A^{\prime}$.

- $c\left(V_{q}^{s}, V_{r}^{s}\right)=\frac{|q-r|}{n_{s}-1}$ for all $q, r \in\left\{0,1, \ldots, n_{s+1}\right\}, s \in\{1, \ldots, P\}$,

- $c\left(V_{q}^{s}, V_{r}^{t}\right)=\frac{1}{n_{s}-1}+\frac{1}{n_{t}-1}+\left|\frac{q-1}{n_{s}-1}-\frac{r-1}{n_{t}-1}\right|$ for all $s \neq t \in\{1, \ldots, P\}$, $q \in\left\{1, \ldots, n_{s}\right\}, r \in\left\{1, \ldots, n_{t}\right\}$.

and $b_{i j}=0$ otherwise. The PB inequality is then

$$
\sum_{(i, j) \in A} b_{i j} x_{i j} \geq 1+\sum_{s=1}^{P} \frac{n_{s}+1}{n_{s}-1}+B .
$$

The right hand side of $\mathrm{PB}$ inequality (9) can also be written as

$$
\sum_{(i, j) \in A} b_{i j} x_{i j} \geq B+\sum_{s=1}^{P} \frac{2 n_{s}}{n_{s}-1}-P+1 .
$$

The validity of the PB inequalities for the DGRP is based on the fact that all the DGRP solutions must traverse all the required arcs in $\left(M_{A}: M_{Z}\right)$ and visit all the vertex sets $M_{q}^{s}$. Consider now a TOARP solution $\left(x^{1}, y^{1}, x^{2}, y^{2}, \ldots, x^{K}, y^{K}\right)$. Note that a given vehicle $k \in\{1, \ldots, K\}$ is not obliged neither to traverse all the required arcs in $\left(M_{A}: M_{Z}\right)$ nor to visit all the vertex sets $M_{q}^{s}$. Hence, only replacing each variable $x_{i j}$ in the PB inequality (10) by the corresponding variable $x_{i j}^{k}$ is not enough to obtain a valid inequality for $\mathcal{P}$. Variables $y_{i j}^{k}$ are also needed in order to know which required arcs in $\left(M_{A}: M_{Z}\right)$ are traversed and which vertex sets $M_{q}^{s}$ are visited by vehicle $k$. This leads to the disaggregate PB inequalities, corresponding to a vehicle $k \in\{1, \ldots, K\}$, that we describe next. 


\begin{tabular}{|c|c|c|c|c|c|c|c|c|}
\hline$x^{1}$ & 2 & $x^{x^{2}}$ & $y_{A_{R}}^{1}$ & $y_{A_{R}}^{2}$ & $y_{A_{R}}^{3}$ & $y_{A^{\prime}}^{1}$ & $y_{A^{\prime}}^{2}$ & $y_{A^{\prime}}^{3}$ \\
\hline $\begin{array}{c}z_{1} \\
\vdots \\
z_{m}\end{array}$ & 0 & 0 & 1 & 0 & 0 & $\begin{array}{cc}1 & \\
\vdots & 0 \\
1 & 0\end{array}$ & 0 & 0 \\
\hline 0 & $\begin{array}{c}z_{1} \\
\vdots \\
z_{m}\end{array}$ & 0 & 0 & 1 & 0 & 0 & $\begin{array}{cc}1 & \\
\vdots & 0 \\
1 & 0\end{array}$ & 0 \\
\hline 0 & 0 & $\begin{array}{c}z_{1} \\
\vdots \\
z_{m}\end{array}$ & 0 & 0 & 1 & 0 & 0 & $\begin{array}{cc}1 & \\
\vdots & 0 \\
1 & \\
\end{array}$ \\
\hline $\begin{array}{c}z_{1} \\
\vdots \\
z_{1}\end{array}$ & $\begin{array}{c}z_{1} \\
\vdots \\
z_{1}\end{array}$ & 0 & I & 1-I & 0 & $\begin{array}{ll}1 & \\
\vdots & 0 \\
1 & \end{array}$ & 0 & 0 \\
\hline 0 & $\begin{array}{c}z_{1} \\
\vdots \\
z_{1}\end{array}$ & $\begin{array}{c}z_{1} \\
\vdots \\
z_{1}\end{array}$ & 0 & I & 1-I & 0 & $\begin{array}{ll}1 & \\
\vdots & 0 \\
1 & 0\end{array}$ & 0 \\
\hline$z_{1}$ & $z_{1}$ & $z_{1}$ & $\begin{array}{lll}10 \ldots 0 \\
10\end{array}$ & $\begin{array}{llllll}0 & 1 & \ldots & 1\end{array}$ & 0 & $10 \ldots 0$ & $\begin{array}{c}0 \\
0\end{array}$ & 0 \\
\hline$\frac{z_{1}}{z_{1}}$ & $\begin{array}{l}z_{1} \\
z_{1}\end{array}$ & $\begin{array}{l}z_{1} \\
z_{1}\end{array}$ & $\frac{10 \ldots 0}{10 \ldots 0}$ & 0 & 0 & 0 & 0 & $\frac{0}{10 \ldots 0}$ \\
\hline $\begin{array}{c}z_{1} \\
\vdots \\
z_{1}\end{array}$ & 0 & 0 & 1 & 0 & 0 & $\begin{array}{ll}1 & \\
\vdots & \mathrm{I} \\
1 & \end{array}$ & 0 & 0 \\
\hline 0 & $\begin{array}{c}z_{1} \\
\vdots \\
z_{1} \\
\end{array}$ & 0 & 0 & 1 & 0 & 0 & $\begin{array}{ll} & \\
\vdots & I \\
1 & \\
\end{array}$ & 0 \\
\hline 0 & 0 & $\begin{array}{c}z_{1} \\
\vdots \\
z_{1}\end{array}$ & 0 & 0 & 1 & 0 & 0 & $\begin{array}{ll}1 & \\
\vdots & \text { I } \\
1 & \\
\end{array}$ \\
\hline
\end{tabular}

Figure 5: TOARP solutions satisfying $y_{i j}^{1}+y_{i j}^{2}+y_{i j}^{3}=1$, for $(i, j) \in A^{\prime}$

Given a partition $\left\{M_{A}, M_{Z},\left\{M_{q}^{s}\right\}_{q=1, \ldots, n_{s}}^{s=1, \ldots, P}\right\}$ of $V$, let $F \subset\left(M_{A}: M_{Z}\right) \cap\left(A_{R} \cup A^{\prime}\right)$ be such that $P+|F| \geq 3$ is an odd number and, for each $s=1, \ldots, P$, let $H^{s}=\left\{a_{1}^{s}, a_{2}^{s}, \ldots, a_{n_{s}}^{s}\right\} \subset$ $A_{R} \cup A^{\prime}$ be such that each $a_{q}^{s} \in A_{R}\left(M_{q}^{s}\right) \cup A^{\prime}\left(M_{q}^{s}\right)$. Note that if vehicle $k$ services the arc $a_{q}^{s}$, i.e., if $y_{a_{q}^{s}}^{k}=1$, then vehicle $k$ has to visit node $M_{q}^{s}$. On the other hand, if the depot $1 \notin M_{A} \cup M_{Z}$ but, say, $1 \in M_{r}^{t}$, then vehicle $k$ is forced to visit set $M_{r}^{t}$ although it does not service the $\operatorname{arc} a_{r}^{t}$. For each vehicle $k$ we call disaggregate $P B$ inequality:

$$
\sum_{(i, j) \in A} b_{i j} x_{i j}^{k} \geq\left(2 y^{k}(F)-|F|\right)+\sum_{s=1}^{P} \frac{2}{n_{s}-1} y^{k}\left(H_{s}\right)-P+1, \quad \text { if } 1 \in M_{A} \cup M_{Z},
$$

and, if for example $1 \in M_{r}^{t}$, after redefining $H_{t}=\left\{a_{1}^{t}, a_{2}^{t}, \ldots, a_{n_{t}}^{t}\right\} \backslash\left\{a_{r}^{t}\right\}$ :

$$
\sum_{(i, j) \in A} b_{i j} x_{i j}^{k} \geq\left(2 y^{k}(F)-|F|\right)+\sum_{s=1}^{P} \frac{2}{n_{s}-1} y^{k}\left(H_{s}\right)+\frac{2}{n_{t}-1}-P+1 .
$$

We also consider PB inequalities related to a subset of vehicles. For a subset of vehicles $\Omega \subset\{1, \ldots, K\}$ we call $\Omega$-aggregate $P B$ inequalities:

$\sum_{(i, j) \in A} b_{i j} \sum_{k \in \Omega} x_{i j}^{k} \geq\left(2 \sum_{k \in \Omega} y^{k}(F)-|F|\right)+\sum_{s=1}^{P} \frac{2}{n_{s}-1} \sum_{k \in \Omega} y^{k}\left(H_{s}\right)-P+1, \quad$ if $\quad 1 \in M_{A} \cup M_{Z}$, 


\begin{tabular}{|c|c|c|c|c|c|c|c|c|c|}
\hline$x^{1}$ & $x^{2}$ & $x^{3}$ & $y_{A_{E}}^{1}$ & $y_{A_{R}}^{2}$ & $y_{A_{R}}^{3}$ & $\begin{array}{ll}a & y_{A}^{1} \\
\end{array}$ & $a$ & $y_{A^{\prime}}^{2}$ & $\begin{array}{r}y_{A^{\prime}}^{3} \\
\end{array}$ \\
\hline $\begin{array}{c}w_{1} \\
\vdots \\
w_{m}\end{array}$ & $\begin{array}{c}z_{1} \\
\vdots \\
z_{1}\end{array}$ & 0 & 0 & 1 & 0 & $\begin{array}{cc}1 & \\
\vdots & 0 \\
1 & \end{array}$ & $\begin{array}{l}0 \\
\vdots \\
0 \\
0\end{array}$ & 1 & 0 \\
\hline 0 & $\begin{array}{c}z_{1} \\
\vdots \\
z_{m} \\
\end{array}$ & 0 & 0 & 1 & 0 & 0 & & 1 & 0 \\
\hline 0 & 0 & $\begin{array}{c}z_{1} \\
\vdots \\
z_{m}\end{array}$ & 0 & 0 & 1 & 0 & & 0 & 0 \\
\hline $\begin{array}{c}w_{i} \\
\vdots \\
w_{r}\end{array}$ & $\begin{array}{c}z_{1} \\
\vdots \\
z_{1} \\
\end{array}$ & 0 & 0 & 1 & 0 & $\begin{array}{ll}1 & \\
\vdots & I \\
1 & \\
\end{array}$ & \begin{tabular}{|l|}
0 \\
$\vdots$ \\
0 \\
\end{tabular} & $1-\mathrm{I}$ & 0 \\
\hline $\begin{array}{c}w_{j} \\
\vdots \\
w_{s}\end{array}$ & $\begin{array}{c}z_{1} \\
\vdots \\
z_{1}\end{array}$ & 0 & I & 1-I & 0 & $\begin{array}{ll}1 & \\
\vdots & 0 \\
1 & \\
\end{array}$ & \begin{tabular}{|l}
0 \\
$\vdots$ \\
0 \\
\end{tabular} & 1 & 0 \\
\hline 0 & $\begin{array}{c}z_{1} \\
\vdots \\
z_{1}\end{array}$ & $\begin{array}{c}z_{1} \\
\vdots \\
z_{1}\end{array}$ & 0 & 0 & 1 & 0 & & I & $1-\mathrm{I}$ \\
\hline 0 & $\begin{array}{c} \\
z_{1} \\
\vdots \\
z_{1} \\
\end{array}$ & $\begin{array}{c}z_{1} \\
\vdots \\
z_{1} \\
\end{array}$ & 0 & I & 1-I & 0 & & 0 & 1 \\
\hline 0 & 0 & $\begin{array}{c}z_{1} \\
\vdots\end{array}$ & 0 & 0 & 1 & 0 & & 0 & I \\
\hline$w_{i}$ & $z_{1}$ & $z_{1}$ & 0 & 1 & 0 & $110 \ldots$ & & $1 \ldots 1$ & 0 \\
\hline
\end{tabular}

Figure 6: TOARP solutions satisfying $\sum_{i \in V \backslash S, j \in S} x_{i j}^{1}=y_{a}^{1}$ for $a \in A^{\prime}$.

and, if $1 \in M_{r}^{t}$, after redefining $H_{t}=\left\{a_{1}^{t}, a_{2}^{t}, \ldots, a_{n_{t}}^{t}\right\} \backslash\left\{a_{r}^{t}\right\}$, :

$$
\sum_{(i, j) \in A} b_{i j} \sum_{k \in \Omega} x_{i j}^{k} \geq\left(2 \sum_{k \in \Omega} y^{k}(F)-|F|\right)+\sum_{s=1}^{P} \frac{2}{n_{s}-1} \sum_{k \in \Omega} y^{k}\left(H_{s}\right)+\frac{2}{n_{t}-1}-P+1 .
$$

Theorem 7 Disaggregate $P B$ inequalities (11) and (12) and $\Omega$-aggregate $P B$ inequalities (13) and (14) are valid for $\mathcal{P}$.

Proof: We will prove it for the disaggregate PB inequalities (11) with the depot $1 \in M_{A} \cup M_{Z}$ and corresponding, for example, to the vehicle $k=1$. The proof in other cases is similar. Let us call

$$
b_{0}=|F|+\sum_{s=1}^{P} \frac{2 n_{s}}{n_{s}-1}-P+1=|F|+\sum_{s=1}^{P} \frac{n_{s}+1}{n_{s}-1}+1,
$$

that is, the right-hand side of the PB inequality (10) for the DGRP. We distinguish several cases.

Let $\left(x^{1}, y^{1}, x^{2}, y^{2}, \ldots, x^{K}, y^{K}\right)$ be a TOARP solution such that vehicle 1 services all the arcs in $F \cup H$. Thus, vehicle 1 traverses all the arcs in $F$ and visits all the sets $M_{q}^{s}$. Given that the PB inequality (10) is valid for the DGRP, vector $x^{1}$ satisfies $\sum_{(i, j) \in A} b_{i j} x_{i j}^{1} \geq b_{0}$. On the other hand, if vehicle 1 services all the arcs in $F \cup H$, then $y^{1}(F)=|F|$ and $y^{1}\left(H_{s}\right)=n_{s}$ 


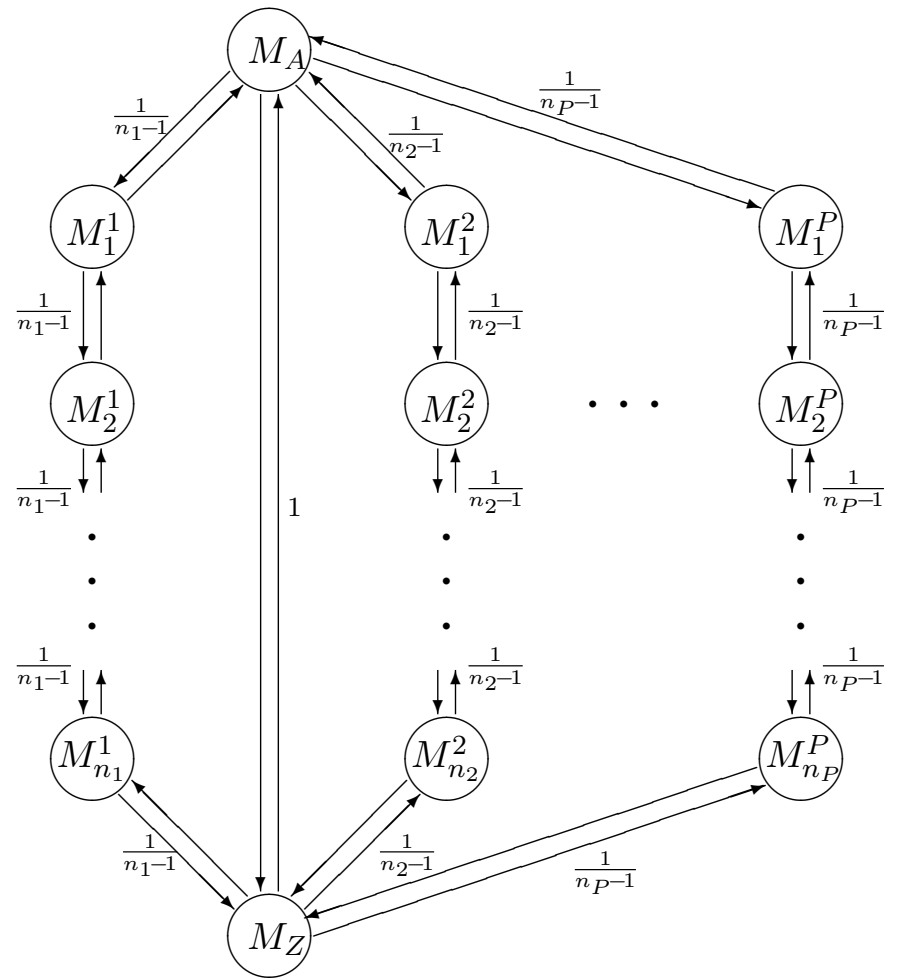

Figure 7: Path-Bridge configuration.

for all $s=1, \ldots, P$. In this case, the right-hand side of the disaggregate PB inequalities (11) takes the value

$$
\left(2 y^{k}(F)-|F|\right)+\sum_{s=1}^{P} \frac{2}{n_{s}-1} y^{k}\left(H_{s}\right)-P+1=(2|F|-|F|)+\sum_{s=1}^{P} \frac{2 n_{s}}{n_{s}-1}-P+1=b_{0},
$$

and hence the TOARP solution satisfies inequality (11).

Let $\left(x^{1}, y^{1}, x^{2}, y^{2}, \ldots, x^{K}, y^{K}\right)$ be a TOARP solution such that vehicle 1 services all the arcs in $F \cup H$ except an arc $f \in F$. Therefore, vehicle 1 traverses $|F|-1 \operatorname{arcs}$ in $\left(M_{A}: M_{Z}\right)$ and visits all the sets $M_{q}^{s}$. Given that $|F|-1+P$ is an even number, the way of doing so minimizing the left-hand side of the constraint is to traverse the $P$ paths once, with value $\sum_{s=1}^{P} \frac{n_{s}+1}{n_{s}-1}$, and the $|F|-1 \operatorname{arcs}$ in $\left(M_{A}: M_{Z}\right)$, with value $|F|-1$. Hence, vector $x^{1}$ satisfies $\sum_{(i, j) \in A} b_{i j} x_{i j}^{1} \geq b_{0}-2$. On the other hand, vector $y^{1}$ satisfies $y^{1}(F)=|F|-1$ and $y^{1}\left(H_{s}\right)=n_{s}$ for all $s$ in this case, and the right-hand side of the disaggregate PB inequalities (11) takes the value

$$
(2|F|-2-|F|)+\sum_{s=1}^{P} \frac{2 n_{s}}{n_{s}-1}-P+1=b_{0}-2,
$$

and hence the TOARP solution satisfies inequality (11).

Let $\left(x^{1}, y^{1}, x^{2}, y^{2}, \ldots, x^{K}, y^{K}\right)$ be a TOARP solution such that vehicle 1 services all the arcs in $F \cup H$ except an $\operatorname{arc} a_{j}^{t} \in H_{t}$. Therefore, vehicle 1 traverses all the arcs in $F$ and 
visits all the sets $M_{q}^{s}$ except set $M_{j}^{t}$ in the path $t$. The way of doing this minimizing the left-hand side of the constraint, with value $b_{0}-\frac{2}{n_{t}-1}$, is to traverse the other $P-1$ paths once, the $|F| \operatorname{arcs}$ in $\left(M_{A}: M_{Z}\right)$ and the path $t$ twice, except for the arcs incident with node $M_{j}^{t}$. Hence, vector $x^{1}$ satisfies $\sum_{(i, j) \in A} b_{i j} x_{i j}^{1} \geq b_{0}-\frac{2}{n_{t}-1}$. On the other hand, vector $y^{1}$ satisfies $y^{1}(F)=|F|$ and $y^{1}\left(H_{s}\right)=n_{s}$ for all $s, s \neq t$, and, in this case, $y^{1}\left(H_{t}\right)=n_{t}-1$. Thus, the right-hand side of the disaggregate $\mathrm{PB}$ inequalities (11) takes the value

$$
(2|F|-|F|)+\sum_{s=1}^{P} \frac{2 n_{s}}{n_{s}-1}-\frac{2}{n_{t}-1}-P+1=b_{0}-\frac{2}{n_{t}-1},
$$

and hence the TOARP solution satisfies inequality (11).

In the same way, it can be seen that the inequality (11) is satisfied by TOARP solutions in which vehicle 1 services any other number of arcs in $F \cup H$.

\subsubsection{2-PB and $n$-regular PB inequalities}

We present here the special cases of $\mathrm{PB}$ inequalities in which $P=2$, which are the only ones that are separated in the branch-and-cut algorithm, and the regular ones, in which all the paths are of the same length.

If $P=2$, the 2-PB inequality can be expressed with integer coefficients by multiplying it by $\left(n_{1}-1\right)\left(n_{2}-1\right)$. For example, inequalities (11) with $P=2$ can be written as:

$$
\begin{aligned}
& \left(n_{1}-1\right)\left(n_{2}-1\right) \sum_{(i, j) \in A} b_{i j} x_{i j}^{k} \geq\left(n_{1}-1\right)\left(n_{2}-1\right)\left(2 y^{k}(F)-|F|\right)+ \\
& \quad+2\left(n_{2}-1\right) y^{k}\left(H_{1}\right)+2\left(n_{1}-1\right) y^{k}\left(H_{2}\right)-\left(n_{1}-1\right)\left(n_{2}-1\right) .
\end{aligned}
$$

On the other hand, a PB inequality is called $n$-regular if all the $P$ paths are of the same length $n$. In this case, by multiplying it by $n-1$, inequalities (11) to (14) can be rewritten in an easier way. For example, we can define $H=H_{1} \cup \cdots \cup H_{P}$ and inequalities (11) can be written as:

$$
(n-1) \sum_{(i, j) \in A} b_{i j} x_{i j}^{k} \geq(n-1)\left(2 y^{k}(F)-|F|\right)+2 y^{k}(H)-(n-1)(P-1) .
$$

Note that coefficients in the regular case, $(n-1) b_{i j}$, are also simpler: variables corresponding to arcs in $\left(V_{q}^{s}, V_{r}^{s}\right)$ have a coefficient equal to $|q-r|$ and those corresponding to $\operatorname{arcs}\left(V_{q}^{s}, V_{r}^{t}\right), \quad s \neq t, \quad q, r \in\{1,2, \ldots, n\}$, now have a coefficient equal to their 'difference in level' plus two, $|i-j|+2$.

\subsubsection{K-C inequalities}

$\mathrm{K}-\mathrm{C}$ inequalities are well-known families of facet-inducing inequalities for other Arc Routing Problems. The name of this family of inequalities is motivated by the number of sets into which $V$ is partitioned, which is usually denoted by $K$. To avoid confusion with the number of vehicles, in what follows we use the letter $Q$ instead. A K-C inequality can be obtained as a particular case of $\mathrm{PB}$ inequalities with $P=1$. It is defined by a partition of $V$ into $Q+1$ 
sets $\left\{M_{0}, M_{1}, M_{2}, \ldots, M_{Q-1}, M_{Q}\right\}$. Hence $M_{A}=M_{0}, M_{Z}=M_{Q}$ and $n=Q-1$. Given that it is a regular $\mathrm{PB}$, after multiplying by $n-1=Q-2$, we call disaggregate $K$ - $C$ inequality associated with vehicle $k$ :

$$
\sum_{(i, j) \in A}(Q-2) b_{i j} x_{i j}^{k} \geq(Q-2)\left(2 y^{k}(F)-|F|\right)+2 y^{k}(H), \quad \text { if } 1 \in M_{0} \cup M_{Q},
$$

and

$$
\sum_{(i, j) \in A}(Q-2) b_{i j} x_{i j}^{k} \geq(Q-2)\left(2 y^{k}(F)-|F|\right)+2+2 y^{k}\left(H \backslash\left\{a_{r}\right\}\right), \quad \text { if } 1 \in M_{r}, r \notin\{0, Q\},
$$

where $Q \geq 3, F \subset\left(M_{0}: M_{Q}\right) \cap\left(A_{R} \cup A^{\prime}\right)$ with $|F| \geq 2$ and even, and $H=\left\{a_{1}, \ldots, a_{Q-1}\right\}$ with each $a_{r} \in A_{R}\left(M_{r}\right) \cup A^{\prime}\left(M_{r}\right)$. The coefficients $(Q-2) b_{i j}$ of the variables in the inequality are shown in Figure 8, where each number represents the coefficient of the variable $x_{i j}^{k}$ associated with the traversal of the corresponding arc:

$$
(Q-2) b_{i j}=\left\{\begin{array}{cl}
Q-2 & \text { if }(i, j) \in\left(M_{0}: M_{Q}\right) \\
|r-s| & \text { if }(i, j) \in\left(M_{r}: M_{s}\right),\{r, s\} \neq\{0, Q\} \\
0 & \text { otherwise. }
\end{array}\right.
$$

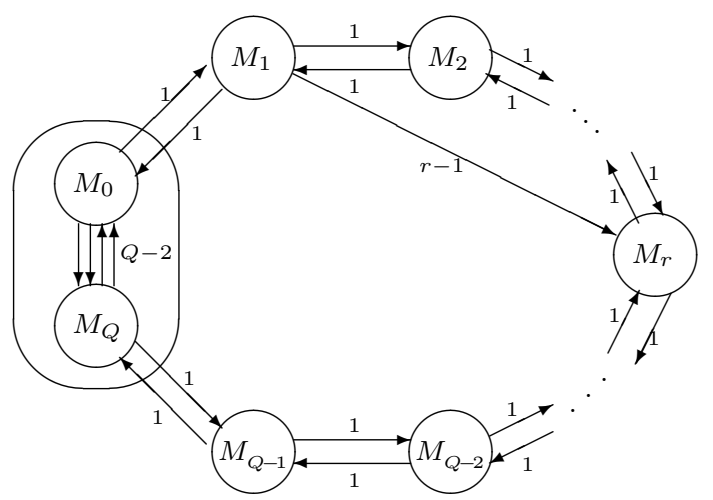

Figure 8: K-C configuration

We can also consider $\mathrm{K}-\mathrm{C}$ inequalities related to a subset of vehicles $\Omega \subset\{1, \ldots, K\}$, called $\Omega$-aggregate $K$ - $C$ inequalities:

$$
\sum_{(i, j) \in A}(Q-2) b_{i j} \sum_{k \in \Omega} x_{i j}^{k} \geq(Q-2)\left(\sum_{k \in \Omega} 2 y^{k}(F)-|F|\right)+\sum_{k \in \Omega} 2 y^{k}(H), \quad \text { if } 1 \in M_{0} \cup M_{Q},
$$

and, if $1 \in M_{r}, r \notin\{0, Q\}$,

$$
\sum_{(i, j) \in A}(Q-2) b_{i j} \sum_{k \in \Omega} x_{i j}^{k} \geq(Q-2)\left(\sum_{k \in \Omega} 2 y^{k}(F)-|F|\right)+2+\sum_{k \in \Omega} 2 y^{k}\left(H \backslash\left\{a_{r}\right\}\right) .
$$




\subsection{Max-time constraints}

In this subsection we introduce some valid inequalities that are derived from inequalities (6) which limit the maximum length of the routes.

Given a set of required arcs and a set of required vertices, remember that the Directed General Routing Problem (DGRP) consists of finding the tour of minimum cost traversing all the required arcs and visiting all the required vertices at least once. Let $F \subset A_{R} \cup A^{\prime}$ and consider the DGRP instance in which the set of required arcs is $F$, the depot is considered a required vertex and the costs of each arc $(i, j)$ is $t_{i j}$. Then, let $z(F)$ represent the optimal value of this DGRP instance. Note that, if $z(F)>T_{\max }$ then we have:

(1) A single vehicle $k$ cannot service all the arcs in $F$ and hence all the feasible solutions satisfy

$$
y^{k}(F) \leq|F|-1 \quad k=1,2, \ldots, K .
$$

(2) If all the arcs in $F$ are serviced, then at least two vehicles must enter $S=V(F)$ and, therefore, all the feasible solutions satisfy

$$
\sum_{k=1}^{K} x^{k}(\delta(S)) \geq 4\left(\sum_{k=1}^{K} y^{k}(F)-|F|+1\right) .
$$

In general, let $p=\left\lceil\frac{z(F)}{T_{\max }}\right\rceil$ and suppose that $z(F)>(p-1) T_{\max }$. Then,

$$
\begin{gathered}
\sum_{k \in \Omega} y^{k}(F) \leq|F|-1 \quad \forall \Omega \subseteq\{1,2, \ldots, K\} \text { such that }|\Omega|=p-1, \quad \text { and } \\
\sum_{k=1}^{K} x^{k}(\delta(S)) \geq 2 p\left(\sum_{k=1}^{K} y^{k}(F)-|F|+1\right)
\end{gathered}
$$

are valid inequalities for the TOARP.

Finally, consider, for instance, that $K=5$ and $p=3$. In this case, when all the arcs in $F$ are serviced, at least 3 vehicles must traverse the cut-set $\delta(S)$. Therefore, from any subset with 4 vehicles, at least 2 vehicles must traverse $\delta(S)$, and from any subset with 3 vehicles, at least one vehicle must traverse it. In general, let $r$ be any integer such that $p-\left(K_{r}\right) \geq 1$ and $r \leq K$. For any subset with $r$ vehicles, $\Omega=\left\{k_{1}, k_{2}, \ldots, k_{r}\right\} \subseteq\{1,2, \ldots, K\}$, the following inequality is satisfied by all the feasible solutions:

$$
\sum_{k \in \Omega} x^{k}(\delta(S)) \geq 2(p-(K-r))\left(\sum_{k=1}^{K} y^{k}(F)-|F|+1\right) .
$$

Inequalities (23) are called max-time $y$-constraints, since they are related to $y$ variables only, while inequalities (24) and (25) are called max-time x-constraints.

\section{The branch-and-cut algorithm}

In this section we present a branch-and-cut algorithm that incorporates separation algorithms for the inequalities described in this paper as well as a lower bound obtained by the metaheuristic algorithm presented in [5]. 


\subsection{Antisymmetry constraints}

Note that given a feasible solution for the TOARP, if the routes associated with two different vehicles are switched, another feasible solution is obtained. Therefore, we have a very large number of equivalent solutions, which hinders the resolution of the problem. To avoid this, we present a set of constraints based on those proposed in [15].

Let us suppose we order the required and optional arcs as $a_{1}, a_{2}, \ldots, a_{K}, \ldots, a_{m}$, where $m=\left|A_{R} \cup A^{\prime}\right|$. The following inequalities help to avoid the symmetry among the vehicles:

- If $a_{1} \in A_{R}$, then we add the equation $y_{a_{1}}^{1}=1$.

- If $a_{1} \in A^{\prime}$, we add the equation $y_{a_{1}}^{2}=0$ and the inequalities $y_{a_{i}}^{2} \leq \sum_{j=1}^{i-1} y_{a_{j}}^{1}, \forall i=2, \ldots, m$.

- In any case, for each $k=3, \ldots, K$, we add the equations $y_{a_{i}}^{k}=0, \forall i \leq k-1$ and the inequalities $y_{a_{i}}^{k} \leq \sum_{j=k-1}^{i-1} y_{a_{j}}^{k-1}, \forall i \geq k$.

\subsection{Initial relaxation}

The initial LP relaxation contains symmetry equations (1), traversing inequalities (3), equations (4) and inequalities (5), ensuring that each required arc is serviced by exactly one vehicle and each optional arc by at most one vehicle, inequalities (6) limiting the length of the routes, trivial inequalities $\left(x_{i j}^{k} \geq 0\right.$ and $\left.0 \leq y_{i j}^{k} \leq 1\right)$, a connectivity inequality (2) associated with each connected component induced by the required arcs, and the set of antisymmetry constraints described above. For this last set of inequalities, the required and optional arcs are ordered based on their distance to the depot. The arc that is farthest from the depot is chosen as the first one in the permutation. The following ones are chosen so that the distance to the depot and those already selected is maximal.

Furthermore, we add the max-time $x$ and $y$-constraints associated with all the subsets of 1,2 , and 3 required and optional arcs, such that more than one vehicle is needed to service them. Finally, if more than one vehicle is needed to service all the required arcs in the graph, we add some more max-time $x$ and $y$-constraints considering the cutsets defined by the depot and some of its adjacent vertices.

\subsection{Separation algorithms}

In this section we present the separation algorithms that have been used to identify the following types of inequalities that are violated by the current LP solution at any iteration of the cutting plane algorithm: disaggregate and $\Omega$-aggregate $\mathrm{K}-\mathrm{C}$ and $\mathrm{PB}$ inequalities, and max-time $x$ and $y$-constraints. Heuristic and exact separation procedures for connectivity inequalities (2) are described in [7].

Given an LP solution $\left(\bar{x}^{1}, \bar{y}^{1}, \bar{x}^{2}, \bar{y}^{2}, \ldots, \bar{x}^{K}, \bar{y}^{K}\right) \in \mathbb{R}^{\left(2|E|+\left|E_{R}\right|\right) K}$, we define the $\Omega$-aggregate vector $\bar{x}^{\Omega}$ associated with a subset $\Omega \subset\{1,2, \ldots, K\}$ of vehicles as $\bar{x}^{\Omega}=\sum_{k \in \Omega} \bar{x}^{k}$ and its associated weighted graph as $\bar{G}^{\Omega}=\left(\bar{V}^{\Omega}, \bar{E}^{\Omega}, \bar{x}^{\Omega}, \bar{y}^{\Omega}\right)$, where $\bar{V}^{\Omega}, \bar{E}^{\Omega}$ are the sets of vertices and edges of the subgraph of $G$ induced by the edges $e \in E$ such that $\bar{x}_{e}^{\Omega}=\bar{x}_{i j}^{\Omega}+\bar{x}_{j i}^{\Omega}>0$, plus the depot, if necessary. Note that both disaggregate and $\Omega$-aggregate inequalities of a given family can be separated with the same procedure, changing only the corresponding input graph $\bar{G}^{\Omega}$. 


\subsubsection{Separation of $\mathrm{K}-\mathrm{C}$ and 2-PB inequalities}

The separation algorithms for disaggregate and $\Omega$-aggregate $\mathrm{K}-\mathrm{C}$ inequalities is similar to that described in [7] for the Min-Max K-vehicles Windy Rural Postman Problem.

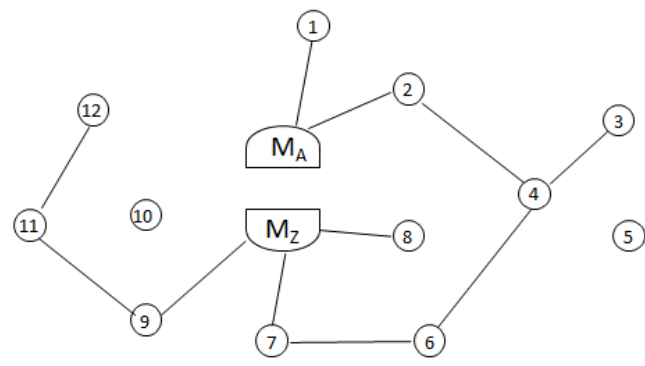

(a)

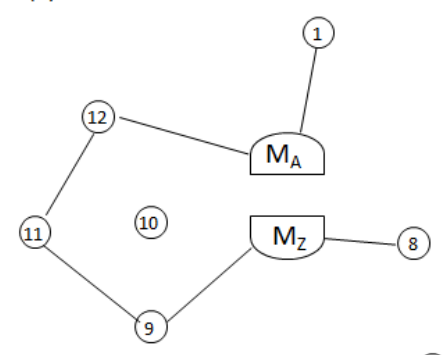

(5)

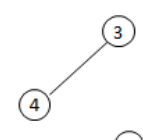

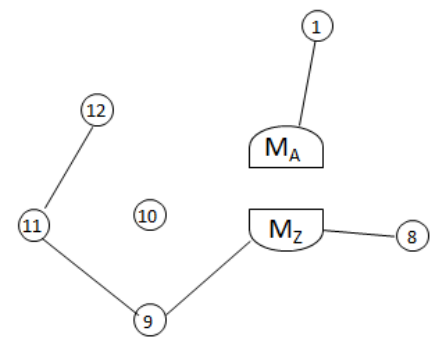

(b)

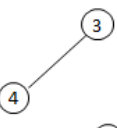

(5)

(c)
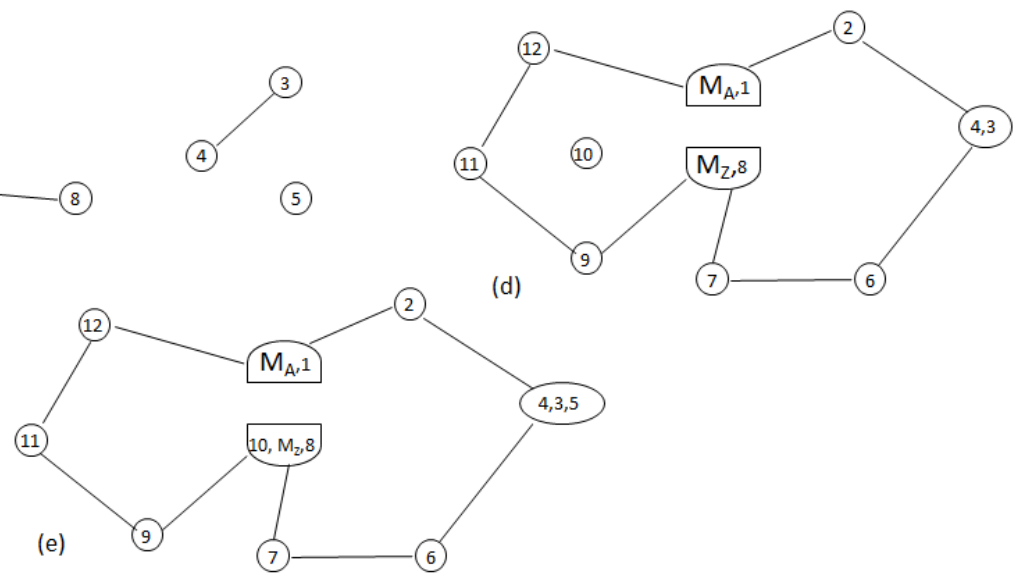

Figure 9: Construction of a PB in the separation algorithm.

A heuristic algorithm based on similar ideas to the ones implemented for the K-C separation has been devised for identifying violated 2-PB inequalities (with only two paths), like those described in Section 4.1.1. More precisely, for a given $\Omega \subset\{1,2, \ldots, K\}$ and its associated weighted graph $\bar{G}^{\Omega}$, the algorithm consists of several phases. Let $C^{1}, \ldots, C^{R}$ be the connected components in $G$ induced by the required and optional arcs.

- For each $C^{i}$, we try to partition it into two parts $M_{A}$ and $M_{Z}$ such that the number of required and optional arcs with $\bar{x}^{\Omega}$ value near 1 is odd, and such that both $M_{A}$ and $M_{Z}$ have at least two arcs in $\bar{G}^{\Omega}$ connecting two other different connected components.

- Given these seeds for $M_{A}$ and $M_{Z}$, consider the graph obtained from $\bar{G}^{\Omega}$ by shrinking the seeds and the remaining $C^{j}$ into a single vertex each. To define the two paths linking $M_{A}$ and $M_{Z}$, we compute a tree by iteratively adding the edge of maximum $\bar{x}^{\Omega}$-weight not forming a cycle and not connecting the seeds (see Figure $9(\mathrm{a})$ ). This tree contains a path joining $M_{A}$ and $M_{Z}$. We remove it and obtain a graph similar to the one depicted in Figure 9(b), where $M_{A}$ and $M_{Z}$ are not connected. We complete the tree with edges of maximum $\bar{x}^{\Omega_{\text {-weight }}}$ until another path linking $M_{A}$ and $M_{Z}$ is obtained (see Figure $9(\mathrm{c})$ ). We iteratively shrink each vertex with degree one in the tree into its unique adjacent vertex in order to obtain two paths joining $M_{A}$ and $M_{Z}$ (see Figure 9(d)). Finally, all the isolated vertices are shrunk into an adjacent vertex in the original graph $G$ (see Figure $9(\mathrm{e})$ ). 
- We now have a 2-PB configuration. Set $F$ is formed of the required and optional arcs in $\left(M_{A}, M_{Z}\right)$ with $\bar{x}^{\Omega}$ value near 1 . For each set $M_{q}^{s}$ not containing the depot, we select the required or optional arc with maximum $\bar{y}^{\Omega}$ weight to define the sets $H_{1}$ and $H_{2}$. The corresponding 2-PB inequality is checked for violation. If it is not violated, we try to shrink the 2-PB configuration by merging some adjacent sets $M_{q}^{s}$.

\subsubsection{Separation of max-time inequalities}

We separate max-time y-constraints (21), corresponding to a single vehicle $k$, and max-time $\mathrm{x}$-constraints (22), corresponding to the set of all the vehicles with procedures similar to those presented in [8].

Briefly, inequalities (21) are separated as follows. Given an LP solution, for each vehicle $k, k=1, \ldots, K$, let $\left\{a_{1}, a_{2}, \ldots, a_{q}\right\}$ be an ordered set of required and optional arcs such that $\bar{y}_{a_{j}}^{k}>0.9$ and $\bar{y}_{a_{1}}^{k} \geq \bar{y}_{a_{2}}^{k} \geq \ldots, \geq \bar{y}_{a_{q}}^{k}$. Then, let $F=\left\{a_{1}, a_{2}, \ldots, a_{f}\right\}$, where $f$ is the maximal number such that $\sum_{a \in F} \bar{y}_{a}^{k}>|F|-0.5$. Iteratively, for each required or optional arc $\bar{a}$ with $0.5<\bar{y}_{\bar{a}}^{k}<0.9$, we consider the set $\bar{F}=F \cup\{\bar{a}\}$. Then the minimum number of vehicles needed to service the arcs in $\bar{F}$ is computed by solving the associated DGRP. If this number is greater than one, we have a violated max-time y-constraint. We also check the inequality (22) corresponding to the set $S^{\prime}$ of vertices incident with the $\operatorname{arcs}$ in $F$ for violation.

The heuristic separation procedure for max-time x-constraints (22) starts by selecting a vertex at random (in such a way that each vertex has a probability of being chosen which is proportional to its distance to the depot). Then a sequence of vertex subsets is created by adding a new vertex iteratively in such a way that $\sum_{k=1}^{K} \bar{x}^{k}(\delta(S))$ is minimum for the resulting subset $S$. For each subset generated, we compute the minimum number of vehicles needed to service all the required and optional arcs incident with vertices in $S$ and the corresponding inequality is checked for violation.

Since computing the minimum number of vehicles needed to service a given set of arcs involves solving a DGRP exactly, we manage two lists containing the studied sets as in [8] in order to minimize the number of DGRP solved.

\subsection{The cutting-plane algorithm}

At each iteration of the cutting plane algorithm the separation procedures are used in the following specific order and the violated inequalities found are added to the LP relaxation:

1. Heuristic separation algorithm for connectivity inequalities (2) for each vehicle $k$.

2. Exact connectivity separation for those vehicles for which the corresponding heuristic has failed.

3. Heuristic algorithms for separating disaggregate and $\Omega$-aggregate $\mathrm{K}-\mathrm{C}$ and 2 -PB inequalities.

4. If no violated inequalities have been found so far, heuristic separation of max-time yconstraints.

5. If no violated inequalities have been found so far, heuristic separation of max-time xconstraints.

As far as the separation of $\Omega$-aggregate $\mathrm{K}-\mathrm{C}$ and 2 -PB inequalities is concerned, we do not know which is the best way to choose the set of vehicles to be aggregated. Given that applying the separation procedures for $\Omega$-aggregate inequalities for each possible combination 
of vehicles is computationally expensive, we proceed as follows. For any pair of vehicles $\left\{k_{1}, k_{2}\right\}$, we apply the separation procedures for $\Omega$-aggregate $\mathrm{K}-\mathrm{C}$ and 2-PB inequalities only when no violated disaggregate inequality of the same type has been found either for $k_{1}$ or $k_{2}$. Moreover, for any subset $\Omega$ with 3 vehicles (if $K \geq 4$ ), we apply the separation procedures for $\Omega$-aggregate inequalities only when no violated disaggregate nor $\Omega$-aggregate inequalities, with $|\Omega|=2$, associated with any of the 3 vehicles has been found. We proceed in a similar way for subsets of 4 vehicles (if $K \geq 5$ ). Additionally, the separation of the $\Omega$-aggregate inequalities is carried out only if the corresponding support graphs $\bar{G}^{k}, k \in \Omega$, are connected.

Since the separation of max-time constraints is computationally expensive, it is only run when no other violated inequalities have been found. Furthermore, when this separation algorithm is executed for ten consecutive iterations in the same node, we proceed to branch. When this happens or no violated inequalities are found by any separation algorithm, we branch by using the Strong Branching strategy [1] implemented in Cplex, giving priority to the variables associated with servicing the required arcs, then to those corresponding to servicing the optional arcs, and finally to $x$ variables.

\section{Computational experiments}

We present here the computational results obtained on a large set of instances based on wellknown ones from the literature. The branch-and-cut procedure has been coded in $\mathrm{C} / \mathrm{C}++$ using the Cplex 12.4 MIP Solver with Concert Technology 2.9 on a single thread of an Intel Core i7 at $3.4 \mathrm{GHz}$ with 16GB RAM. Cplex heuristic algorithms and cut generation were turned off. The optimality gap tolerance was set to zero, and strong branching and the best bound strategies were selected. All the tests were run with a time limit of one hour.

\subsection{Instances}

Since there are no instances of the TOARP in the literature, we have generated TOARP instances from undirected Rural Postman Problem (RPP) ones. Given an RPP instance, we proceed as follows. For each edge $\{i, j\}$, two $\operatorname{arcs}(i, j)$ and $(j, i)$ with the same cost are generated. If the edge is non-required, both arcs are declared non-required too. If it is required, one direction is randomly selected and the corresponding arc is declared required (with probability $p$ ) or optional (with probability $1-p$ ), while the opposite one is declared non-required. The profit associated with the traversal of an optional arc is defined as its cost.

The particular RPP instances we have used here are those proposed by Hertz et al. [16]. In these instances, vertices are points in the Euclidean plane and edge costs are defined by the Euclidean distances. The set of edges is defined according to three different methods. In the first method ( $\mathrm{R}$ class instances) the edges are generated randomly in the plane. The second method ( $G$ class instances) generates a set of edges with cost 1, defining a uniform grid. The third method (D class instances) chooses edges defining a graph where all the vertices have degree 4 . From this set of instances, we have removed the smallest ones. From each one of these $69 \mathrm{RPP}$ instances we have generated 9 TOARP instances corresponding to each combination of the values $0,0.25,0.5$ and $2,3,4$ for parameters $p$ and $K$, respectively. The characteristics of the 69 TOARP instances for each value of parameter $p$ can be seen in Table 1. Each row in this table shows the minimum and maximum number of vertices, arcs, required arcs, and optional arcs for each group of instances.

The value of $T_{\max }$ has been generated for each TOARP instance such that there is a feasible solution servicing all the required arcs, but the optimal solution does not service all 
the optional arcs. The selection of these $T_{\max }$ values is not trivial, since a large value would allow all the profitable arcs to be serviced, making the problem similar to a DRPP with a different objective function. On the other hand, a small value could make the problem infeasible, as some of the required arcs could not be serviced. To overcome this issue, in some cases it has been necessary to solve two instances of the Min-max K-vehicles DRPP with the algorithm proposed in [8]. Given a directed graph, a subset of required arcs, and a fixed number of vehicles $K$, this problem consists of finding a set of $K$ routes servicing all the required arcs such that the length of the longest route is minimal. Given a TOARP instance, two Min-max K-DRPP instances are defined: one in which the required arcs are those in $A_{R}$, and a second one in which they are those in $A_{R} \cup A^{\prime}$. Then the value for $T_{\max }$ is chosen as a number between the costs of the optimal solutions of these two instances.

\begin{tabular}{|c|c|c|c|c|c|c|c|c|c|}
\hline \multirow[b]{2}{*}{ Set } & \multirow[b]{2}{*}{ \# inst } & \multirow[b]{2}{*}{$|V|$} & \multirow[b]{2}{*}{$|A|$} & \multicolumn{2}{|c|}{$p=0$} & \multicolumn{2}{|c|}{$p=0.25$} & \multicolumn{2}{|c|}{$p=0.5$} \\
\hline & & & & $\left|A_{R}\right|$ & $\left|A^{\prime}\right|$ & $\left|A_{R}\right|$ & $\left|A^{\prime}\right|$ & $\left|A_{R}\right|$ & $\left|A^{\prime}\right|$ \\
\hline R30 & 5 & $11-18$ & $42-134$ & 0 & $7-11$ & $1-3$ & 4-9 & $3-6$ & $6-2$ \\
\hline $\mathrm{R} 40$ & 5 & $13-25$ & $68-266$ & 0 & $8-18$ & $3-5$ & $3-15$ & $4-8$ & $1-11$ \\
\hline $\mathrm{R} 50$ & 5 & $19-27$ & $166-296$ & 0 & $13-20$ & $0-7$ & $11-17$ & $4-11$ & $8-10$ \\
\hline D36 & 9 & $17-36$ & $96-270$ & 0 & $10-38$ & $2-10$ & $6-30$ & $6-20$ & $4-23$ \\
\hline D64 & 9 & $37-62$ & 264-482 & 0 & $27-75$ & $4-21$ & $22-54$ & $11-38$ & $15-37$ \\
\hline D100 & 9 & $68-100$ & $544-846$ & 0 & $50-121$ & $9-28$ & $37-95$ & $26-64$ & $20-70$ \\
\hline G36 & 9 & $18-35$ & 54-120 & 0 & $11-35$ & $1-11$ & $7-28$ & $6-18$ & $3-19$ \\
\hline G64 & 9 & $34-62$ & $128-228$ & 0 & $24-68$ & $3-22$ & $20-50$ & $10-15$ & $12-38$ \\
\hline G100 & 9 & $60-100$ & 246-394 & 0 & $41-113$ & $8-25$ & 33-91 & $19-57$ & $20-64$ \\
\hline
\end{tabular}

Table 1: TOARP instance characteristics

The set of $69 \times 3$ instances can be found in http://www.uv.es/corberan, as well as the optimal value, when known, or the best feasible solution known and the upper bound obtained at the root node for each instance and for each number of vehicles.

\subsection{Computational results}

The average computational results obtained on this set of 207 instances for two, three and four vehicles are shown in Tables 2 to 4 .

Each one of these tables contains the results corresponding to a different class of instances: $\mathrm{R}, \mathrm{D}$, and G. These results, grouped by instance size, have been arranged in matrices corresponding to the different combinations of values for the number of vehicles $K$ and parameter $p$. The first row of each block shows the number of instances solved to optimality within the time limit of one hour. The second row gives the average percentage gap between the upper bound at the end of the root node and the optimal value (or the best known solution), while the final gap is shown in the third row. The fourth and fifth rows report the average time and number nodes, respectively, used by the branch-and-cut algorithm for the optimally solved instances.

Overall, the results obtained are very good, especially when considering the great difficulty of this problem, in which the length of the routes is not included in the objective function, whose value is determined exclusively by the profit from the optional arcs serviced.

These results confirm a fact common to the resolution of most multi-vehicle arc routing problems by branch-and-cut algorithms: the increasing difficulty of the problem with the 
number of vehicles. Particularly, we have been capable of solving 204 out of 207 instances with $K=2$ to optimality, 188 for $K=3$, and only 157 for $K=4$. Instances of class $\mathrm{R}$ are easier to solve, mainly because of their smaller size and, we think, the random structure of the graphs and the wide range of the costs of the arcs. If we examine the results corresponding to classes $\mathrm{D}$ and $\mathrm{G}$, it can be seen that the case where $p=0$ is clearly more difficult than the others. The reason for this behavior is that when there are no required arcs, the vehicles have more options for constructing their routes and the solution set is larger. It is interesting to note that this behavior is opposite to the one we have experienced with the metaheuristic algorithm, as explained in [5].

Finally, we would like to note that although some gap values at the root node may not seem very good, this is partly due to the fact that in some instances the profit from (and the cost of) the arcs is quite large and the objective function only takes into account the profit collected.

\begin{tabular}{|c|c|c|c|c|c|c|c|c|c|c|}
\hline & \multicolumn{3}{|c|}{$p=0$} & \multicolumn{3}{|c|}{$p=0.25$} & \multicolumn{3}{|c|}{$p=0.5$} \\
\hline & & R30 & $\mathrm{R} 40$ & $\mathrm{R} 50$ & R30 & $\mathrm{R} 40$ & $\mathrm{R} 50$ & R30 & $\mathrm{R} 40$ & $\mathrm{R} 50$ \\
\hline \multirow{5}{*}{$K=2$} & \# of opt & 5 & 5 & 5 & 5 & 5 & 5 & 5 & 5 & 5 \\
\hline & Gap 0 & 6.2 & 6.7 & 9.5 & 2.0 & 10.2 & 8.2 & 0.0 & 9.2 & 130.3 \\
\hline & Gap & 0.0 & 0.0 & 0.0 & 0.0 & 0.0 & 0.0 & 0.0 & 0.0 & 0.0 \\
\hline & Time & 1.0 & 4.1 & 14.4 & 0.3 & 1.3 & 3.6 & 0.3 & 0.6 & 1.9 \\
\hline & Nodes & 1.4 & 19.0 & 50.2 & 0.0 & 9.0 & 16.8 & 0.0 & 1.6 & 5.8 \\
\hline \multirow{5}{*}{$K=3$} & \# of opt & 5 & 5 & 5 & 5 & 5 & 5 & 5 & 5 & 5 \\
\hline & Gap 0 & 0.9 & 5.6 & 7.4 & 2.3 & 6.8 & 8.6 & 5.3 & 9.4 & 37.1 \\
\hline & Gap & 0.0 & 0.0 & 0.0 & 0.0 & 0.0 & 0.0 & 0.0 & 0.0 & 0.0 \\
\hline & Time & 0.3 & 13.9 & 18.9 & 0.5 & 9.1 & 14.9 & 0.5 & 5.9 & 3.0 \\
\hline & Nodes & 1.0 & 41.0 & 31.6 & 0.8 & 25.8 & 31.6 & 0.0 & 15.4 & 6.6 \\
\hline \multirow{5}{*}{$K=4$} & \# of opt & 5 & 5 & 5 & 5 & 5 & 5 & 5 & 5 & 5 \\
\hline & Gap 0 & 0.0 & 1.3 & 5.6 & 0.0 & 2.0 & 8.4 & 0.0 & 2.1 & 1.7 \\
\hline & Gap & 0.0 & 0.0 & 0.0 & 0.0 & 0.0 & 0.0 & 0.0 & 0.0 & 0.0 \\
\hline & Time & 0.2 & 7.7 & 6.3 & 0.1 & 5.1 & 11.2 & 0.2 & 11.0 & 0.5 \\
\hline & Nodes & 0.0 & 22.0 & 6.8 & 0.0 & 22.8 & 29.8 & 0.0 & 27.0 & 0.2 \\
\hline
\end{tabular}

Table 2: Computational results with R30, R40 and R50 sets of instances

\section{Conclusions}

In this paper we have introduced the Team Orienteering Arc Routing Problem, which is the extension to the arc routing setting of the Team Orienteering Problem. For this problem we have presented an integer programming formulation and studied the associated polyhedron. From this polyhedral description we have devised and implemented a branch-and-cut algorithm producing very good computational results on a large set of benchmark instances.

The TOARP has proven to be a very difficult problem. Being a multi-vehicle problem with constraints limiting the length of the routes of the vehicles, it has the intrinsic difficulty of capacitated arc routing problems. Moreover, it presents an objective function that does not consider the route lengths, but only the profit collected from potential customers, while 


\begin{tabular}{|c|c|c|c|c|c|c|c|c|c|c|}
\hline & \multicolumn{3}{|c|}{$p=0$} & \multicolumn{3}{|c|}{$p=0.25$} & \multicolumn{3}{|c|}{$p=0.5$} \\
\hline & & D36 & D64 & D100 & D36 & D64 & D100 & D36 & D64 & D100 \\
\hline \multirow{5}{*}{$K=2$} & \# of opt & 9 & 9 & 9 & 9 & 9 & 9 & 9 & 9 & 9 \\
\hline & Gap 0 & 4.9 & 2.7 & 0.8 & 5.1 & 2.1 & 0.9 & 10.6 & 2.8 & 2.0 \\
\hline & Gap & 0.0 & 0.0 & 0.0 & 0.0 & 0.0 & 0.0 & 0.0 & 0.0 & 0.0 \\
\hline & Time & 11.3 & 37.4 & 408.2 & 5.7 & 30.6 & 152.4 & 4.6 & 32.7 & 320.8 \\
\hline & Nodes & 34.1 & 169.8 & 438.9 & 33.2 & 192.4 & 261.8 & 21.4 & 138.4 & 407.9 \\
\hline \multirow{5}{*}{$K=3$} & \# of opt & 9 & 9 & 4 & 9 & 9 & 5 & 9 & 9 & 7 \\
\hline & Gap 0 & 7.1 & 8.1 & 10.5 & 4.2 & 5.6 & 9.3 & 24.5 & 11.4 & 14.4 \\
\hline & Gap & 0.0 & 0.0 & 2.2 & 0.0 & 0.0 & 0.7 & 0.0 & 0.0 & 0.7 \\
\hline & Time & 68.2 & 382.6 & 1349.9 & 11.7 & 231.2 & 757.4 & 13.5 & 204.9 & 820.8 \\
\hline & Nodes & 93.7 & 763.3 & 1074.0 & 23.3 & 365.7 & 1336.6 & 28.3 & 461.0 & 1137.2 \\
\hline \multirow{5}{*}{$K=4$} & \# of opt & 9 & 5 & 2 & 9 & 7 & 4 & 9 & 7 & 5 \\
\hline & Gap 0 & 4.7 & 6.7 & 7.9 & 5.6 & 7.8 & 7.8 & 5.4 & 10.3 & 7.2 \\
\hline & Gap & 0.0 & 1.3 & 4.6 & 0.0 & 0.6 & 3.0 & 0.0 & 0.3 & 3.4 \\
\hline & Time & 109.7 & 479.7 & 0.4 & 24.1 & 617.7 & 563.9 & 8.5 & 534.5 & 936.3 \\
\hline & Nodes & 75.1 & 715.0 & 392.2 & 31.0 & 1454.0 & 821.3 & 9.9 & 1655.4 & 589.6 \\
\hline
\end{tabular}

Table 3: Computational results with D36, D64 and D100 sets of instances

\begin{tabular}{|c|c|c|c|c|c|c|c|c|c|c|}
\hline & \multicolumn{3}{|c|}{$p=0$} & \multicolumn{3}{|c|}{$p=0.25$} & \multicolumn{3}{|c|}{$p=0.5$} \\
\hline & & G36 & G64 & G100 & G36 & G64 & G100 & G36 & G64 & G100 \\
\hline \multirow{5}{*}{$K=2$} & \# of opt & 9 & 9 & 8 & 9 & 9 & 8 & 9 & 9 & 8 \\
\hline & Gap 0 & 7.3 & 6.2 & 4.1 & 2.2 & 1.4 & 2.1 & 7.2 & 13.2 & 1.5 \\
\hline & Gap & 0.0 & 0.0 & 0.2 & 0.0 & 0.0 & 0.3 & 0.0 & 0.0 & 0.5 \\
\hline & Time & 4.8 & 7.4 & 48.1 & 0.8 & 1.5 & 10.8 & 1.1 & 2.3 & 8.4 \\
\hline & Nodes & 7.7 & 6.7 & 203.8 & 1.3 & 1.8 & 283.7 & 0.8 & 1.8 & 386.6 \\
\hline \multirow{5}{*}{$K=3$} & \# of opt & 9 & 9 & 5 & 9 & 9 & 6 & 9 & 9 & 8 \\
\hline & Gap 0 & 6.3 & 7.6 & 5.5 & 7.0 & 11.1 & 5.0 & 9.6 & 9.4 & 6.4 \\
\hline & Gap & 0.0 & 0.0 & 1.4 & 0.0 & 0.0 & 0.9 & 0.0 & 0.0 & 0.6 \\
\hline & Time & 87.9 & 384.9 & 950.0 & 30.9 & 72.8 & 138.4 & 13.2 & 74.2 & 800.0 \\
\hline & Nodes & 224.3 & 287.7 & 566.9 & 40.9 & 202.2 & 204.2 & 15.7 & 144.7 & 313.6 \\
\hline \multirow{5}{*}{$K=4$} & \# of opt & 8 & 3 & 1 & 8 & 9 & 4 & 7 & 9 & 6 \\
\hline & Gap 0 & 4.4 & 8.0 & 10.2 & 1.9 & 8.5 & 9.5 & 2.0 & 7.6 & 12.1 \\
\hline & Gap & 0.4 & 4.7 & 8.4 & 0.5 & 0.0 & 5.0 & 2.0 & 0.0 & 7.9 \\
\hline & Time & 118.0 & 32.3 & 0.3 & 19.0 & 164.1 & 498.7 & 0.3 & 121.0 & 439.7 \\
\hline & Nodes & 2310.1 & 752.0 & 585.8 & 2124.9 & 68.7 & 438.6 & 2726.3 & 49.2 & 109.9 \\
\hline
\end{tabular}

Table 4: Computational results with G36, G64 and G100 sets of instances 
the time or length limit constraints consider all the arcs traversed by the vehicles. Despite this, we have been able to solve instances with up to 100 vertices, $800 \operatorname{arcs}$, and 4 vehicles.

Acknowledgments: Ángel Corberán, Isaac Plana and José M. Sanchis wish to thank the Ministerio de Ciencia e Innovación of Spain (project MTM2009-14039-C06-02) for its support.

\section{References}

[1] D. Applegate, R.E. Bixby, V. Chvátal and W. Cook (1995), Finding Cuts in the TSP. Technical report, DIMACS 95-05.

[2] J. Aráoz, E. Fernández and C. Franquesa (2009), The Clustered Price-collecting Arcrouting Problem. Transportation Science 43, 287-300.

[3] J. Aráoz, E. Fernández and O. Meza (2009), Solving the Prize-collecting Rural Postman Problem. European Journal of Operational Research 196, 886-896.

[4] J. Aráoz, E. Fernández and C. Zoltan (2006), Privatized Rural Postman Problems. Computers \& Operations Research 33, 3432-3449.

[5] C. Archetti, Á. Corberán, I. Plana, J.M. Sanchis and M.G. Speranza (2012), A Matheuristic for the Team Orienteering Arc Routing Problem. In preparation.

[6] C. Archetti, D. Feillet, A. Hertz and M.G. Speranza (2010), The Undirected Capacitated Arc Routing Problem with Profits. Computers \& Operations Research 37, 1860-1869.

[7] E. Benavent, Á. Corberán, I. Plana and J.M. Sanchis (2009), Min-Max K-vehicles Windy Rural Postman Problem. Networks 54, 216-226.

[8] E. Benavent, Á. Corberán, I. Plana and J.M. Sanchis (2011), New Facets and an Enhanced Branch-and-Cut for the Min-Max K-vehicles Windy Rural Postman Problem. Networks 58, 255-272.

[9] Á. Corberán, E. Fernández, C. Franquesa and J.M. Sanchis (2011), The Windy Clustered Prize-collecting Problem. Transportation Science 45, 317-334.

[10] Á. Corberán, I. Plana, A. Rodríguez-Chía and J.M. Sanchis (2011), A Branch-and-Cut for the Maximum Benefit Chinese Postman Problem. Mathematical Programming, DOI: 10.1007/s10107-011-0507-6.

[11] Á. Corberán, A. Romero and J.M. Sanchis (2003), The Mixed General Routing Polyhedron. Mathematical Programming 96, 103-137.

[12] G. Cornuéjols, J. Fonlupt and D. Naddef (1985), The Traveling Salesman on a Graph and some Related Integer Polyhedra. Mathematical Programming 33, 1-27.

[13] D. Feillet, P. Dejax and M. Gendreau (2005a), Traveling Salesman Problems with Profits. Transportation Science 39, 188-205.

[14] D. Feillet, P. Dejax and M. Gendreau (2005b), The Profitable Arc Tour Problem: Solution with a Branch-and-Price Algorithm. Transportation Science 39, 539-552. 
[15] G. Ghiani, D. Laganá, G. Laporte and R. Musmanno (2007), A Branch-and-Cut Algorithm for the Undirected Capacitated Arc Routing Problem. Les Cahiers du GERARD, Université de Montréal, G-2007-39.

[16] A. Hertz, G. Laporte and P. Nanchen-Hugo (1999), Improvement Procedures for the Undirected Rural Postman Problem. INFORMS Journal on Computing 1, 53-62.

[17] A.N. Letchford (1997), New Inequalities for the General Routing Problem. European Journal of Operational Research 96, 317-322.

[18] C. Malandraki and M.S. Daskin (1993), The Maximum Benefit Chinese Postman Problem and the Maximum Benefit Traveling Salesman Problem. European Journal of Operational Research 65, 218-234.

[19] W. L. Pearn and W. C. Chiu (2005), Approximate Solutions for the Maximum Benefit Chinese Postman Problem. International Journal of Systems Science 36, 815-822.

[20] W.L. Pearn and K.H. Wang (2003), On the Maximum Benefit Chinese Postman Problem. OMEGA 31, 269-273.

[21] P. Vansteenwegen, W. Souffriau, D. Van Oudheusden (2011), The Orienteering Problem: A survey. European Journal of Operational Research 209, 1-10. 